\title{
Factors associated with successful dietary changes in an energy-reduced Mediterranean diet intervention: a longitudinal analysis in the PREDIMED-Plus trial
}

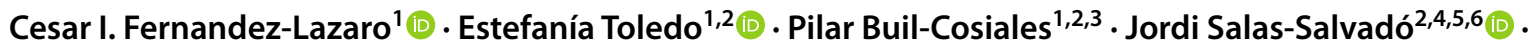 \\ Dolores Corella ${ }^{2,7} \cdot$ Montserrat Fitó ${ }^{2,8} \cdot$ J. Alfredo Martínez ${ }^{2,9,10}$. Ángel M. Alonso-Gómez ${ }^{2,11}$. Julia Wärnberg ${ }^{2,12}$. \\ Jesús Vioque ${ }^{13,14}$ • Dora Romaguera ${ }^{2,15}$. José López-Miranda2,16 Ramon Estruch $^{2,17}$ • Francisco J. Tinahones ${ }^{2,18}$. \\ José Lapetra ${ }^{2,19}$. Luís Serra-Majem ${ }^{2,20}$. Aurora Bueno-Cavanillas ${ }^{13,21}$. Josep A. Tur ${ }^{2,22}$. Vicente Martín Sánchez ${ }^{13,23}$.

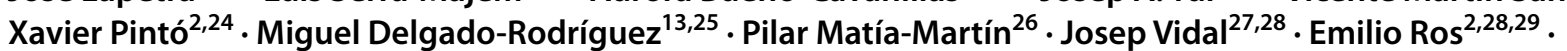 \\ Clotilde Vázquez $^{30}$ - Lidia Daimiel ${ }^{31}$ - Beatriz SanJulián ${ }^{1}$. Jesús F. García-Gavilán ${ }^{2,4}$ • Jose V. Sorlí2,7 Olga Castañer $^{2,8}$. \\ M. Ángeles Zulet ${ }^{2,10}$ • Lucas Tojal-Sierra ${ }^{2,11}$. Napoleón Pérez-Farinós ${ }^{2,12}$ - Alejandro Oncina-Canovas ${ }^{14}$. \\ Manuel Moñino ${ }^{2,15} \cdot$ Antonio Garcia-Rios ${ }^{2,16} \cdot$ Emilio Sacanella $^{2,17} \cdot$ Rosa M. Bernal-Lopez $^{2,18}$. \\ José Manuel Santos-Lozano ${ }^{2,19} \cdot$ Zenaida Vázquez-Ruiz', ${ }^{1,2}$ Jananee Muralidharan ${ }^{2,4}$. Carolina Ortega-Azorín ${ }^{2,7}$. \\ Alberto Goday $^{2,8}$. Cristina Razquin ${ }^{1,2} \cdot$ Leire Goicolea-Güemez ${ }^{2,11} \cdot$ Miguel Ruiz-Canela $^{1,2} \cdot$ Nerea Becerra-Tomás ${ }^{2,4,6}$. \\ Helmut Schröder ${ }^{2,8} \cdot$ Miguel A. Martínez González ${ }^{1,2,32}$. $\cdot$ for the PREDIMED-Plus investigators
}

Received: 2 May 2021 / Accepted: 1 October 2021 / Published online: 30 November 2021

(c) The Author(s) 2021

\begin{abstract}
Purpose Long-term nutrition trials may fail to respond to their original hypotheses if participants do not comply with the intended dietary intervention. We aimed to identify baseline factors associated with successful dietary changes towards an energy-reduced Mediterranean diet (MedDiet) in the PREDIMED-Plus randomized trial.

Methods Longitudinal analysis of 2985 participants (Spanish overweight/obese older adults with metabolic syndrome) randomized to the active intervention arm of the PREDIMED-Plus trial. Dietary changes were assessed with a 17-item energyreduced MedDiet questionnaire after 6 and 12 months of follow-up. Successful compliance was defined as dietary changes from baseline of $\geq 5$ points for participants with baseline scores $<13$ points or any increase if baseline score was $\geq 13$ points. We conducted crude and adjusted multivariable logistic regression models to identify baseline factors related to compliance. Results Consistent factors independently associated with successful dietary change at both 6 and 12 months were high baseline perceived self-efficacy in modifying diet $\left(\mathrm{OR}_{6-\text { month }_{1}}: 1.51,95 \%\right.$ CI 1.25-1.83; OR ${ }_{12 \text {-month }}: 1.66,95 \%$ CI 1.37-2.01), higher baseline fiber intake $\left(\mathrm{OR}_{6 \text {-month }}: 1.62,95 \%\right.$ CI 1.07-2.46; OR 12-month $_{1.62,95 \%}$ CI 1.07-2.45), having $>3$ chronic

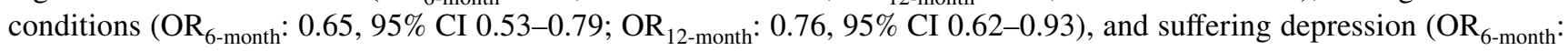
$0.80,95 \%$ CI $0.64-0.99$ OR $_{12-\text { month }}: 0.71,95 \%$ CI $\left.0.57-0.88\right)$.

Conclusion Our results suggested that recruitment of individuals with high perceived self-efficacy to dietary change, and those who initially follow diets relatively richer in fiber may lead to greater changes in nutritional recommendations. Participants with multiple chronic conditions, specifically depression, should receive specific tailored interventions.

Trial registration ISRCTN registry 89898870, 24th July 2014 retrospectively registered http://www.isrctn.com/ISRCTN8989 8870 .
\end{abstract}

Keywords PREDIMED-Plus $\cdot$ Dietary change $\cdot$ Factors $\cdot$ Dietary adherence $\cdot$ Mediterranean diet $\cdot$ Randomized controlled trials

Miguel A. Martínez González

mamartinez@unav.es

Extended author information available on the last page of the article 


\section{Introduction}

Randomized controlled trials (RCTs) are considered the gold standard for clinical nutrition research. They offer scientific evidence of the highest quality level to infer causality of the health effects of diet interventions [1]. However, an important potential limitation in intervention trials occurs when participants do not comply with the intended dietary intervention, which may lead to worthless results [2].

In long-term randomized nutritional trials, participants require a high level of commitment to modify their diet. When participants do not sufficiently adherence to their assigned intervention, no substantial between-group contrast may be attained, and the magnitude of dietary effects can be considerably reduced. Consequently, the results of such trials render misleading results or null findings. As such, after 8 years of follow-up in the Randomized Controlled Dietary Modification Trial of the Women's Health Initiative (WHI), a low-fat dietary intervention did not significantly reduce the risk of breast cancer, total cancer, coronary heart disease, certain chronic diseases, and total mortality when compared with a usual high-fat diet [3, 4]. However, the trial failed to achieve the $14 \%$ intended fat difference between the intervention and control groups; in fact, only $8 \%$ of energy fat reduction was achieved. In addition, further challenges to compliance with interventions on low-carbohydrate diets have been reported [5]. Lack of adherence was also reported in the Multiple Risk Factor Intervention Trial (MRFIT), an intervention that intended to obtain reductions in serum cholesterol (with diet), smoking cessation, and treatment of hypertension [6]. Contrasts in dietary changes between the control and intervention groups in the MRFIT were insufficient as to observe significant differences on cardiovascular disease (CVD) [7]. Even in the PREDIMED trial, which successfully demonstrated strong evidence on the role of the Mediterranean diet (MedDiet) on primary prevention of CVD [8], stronger beneficial effects would have been expected if participants had greater adhered to the intervention diet and the low-fat diet as reported in complementary per-protocol analyses. In fact, the per-protocol analyses suggested a much stronger risk reduction in the MedDiet groups compared to the control group than the intentionto-treat analysis.

Total exclusion in advance of overall non-compliant participants in dietary interventions is unattainable as well as it is unrealistic to believe that the original standard during RCTs can be maintained in normal life. Moreover, it is well known that RCTs usually attain only a suboptimal external validity. However, RCTs represent the gold standard for causal inference and recruiting a population that theoretically may help to maximize compliance with the targeted dietary interventions will make the trial more feasible and will potentially ensure sufficient exposure contrast, contributing to make the RCT more informative from a causal inference point of view. Otherwise, costly, longterm trials may continue failing to respond to its original hypothesis. Determining which patients' and which design components maximize compliance can help investigators identify the most appropriate candidates and modifiable study features that are amenable to be redesigned. Limited knowledge exists on participants' characteristics which may predict compliance to an intervention fostering the adherence to a healthy dietary pattern such as the MedDiet [9-15]. Most of this research has been limited to crosssectional studies [11-15], with few longitudinal studies examining factors that predict dietary change in clinical trials $[9,10]$. Thus, we aimed to identify factors of compliance to an energy-reduced MedDiet (erMedDiet) after 6 and 12 months of follow-up in the PREDIMED-Plus (PREvención con DIeta MEDiterránea Plus) randomized trial, a 6-year parallel-group, multicenter weight-loss lifestyle intervention program.

\section{Methods}

\section{Study design and participants}

The present study is a longitudinal analysis restricted only to the intervention group of the PREDIMED-Plus trial. The study design and procedures of PREDIMED-Plus have been described in detail $[16,17]$. In brief, it assesses the effect of an intensive lifestyle weight-loss intervention on the primary prevention of hard cardiovascular events. The intervention consists of an erMedDiet together with physical activity promotion and behavioral support for specific weight-loss goals on primary prevention of CVD events. More specifically, participants in the intervention group $(n=3406)$ regularly received individual motivational interviews and monthly phone calls, and attended group sessions in which trained dietitians encourage them to adopt suitable dietary and lifestyle changes. The erMedDiet intervention targeted a reduction of approximately $30 \%$ of estimated energy requirements, which represented a reduction goal of approximately $600 \mathrm{kcal} /$ day $[16,17]$. Moreover, the erMedDiet aimed to promote a better overall quality of the diet through the limitation of certain foods such as sugar-sweetened beverages, red and processed meats, butter and cream, added sugars, sweets and pastries, and refined cereals, including white bread, in favor of whole grains. Physical activity promotion included a face-to-face educational program [18] aimed to gradually increase participants' aerobic physical activity levels to meet, at least, the World Health Organization (WHO) guidelines according to the participants' age and 
health status [19]. Recommendations of physical activities also included static exercises to improve resistance, strength, flexibility, and balance. On the other hand, participants in the control group $(n=3468)$ were encouraged to follow an unrestricted energy MedDiet, had biannual educational sessions on the traditional MedDiet with ad libitum caloric intake, and received usual care of general lifestyle recommendations.

Potential candidates to participate in the PREDIMEDPlus trial were overweight/obese [body mass index (BMI) $\left.27-40 \mathrm{~kg} / \mathrm{m}^{2}\right]$ males (aged 55-75 years of age) and females (60-75 years of age), with metabolic syndrome [20], and free of CVD at enrollment. The recruitment of participants of the PREDIMED-Plus took place from September 2013 until October 2016 in 23 Spanish centers. After completing a 4-week run-in period after the initial screening visit, participants were allocated in a 1:1 ratio (either the intervention or control arm) using a computer-generated random allocation sequence stratified by sex, age $(<65,65-70,>70$ years of age), and center, which was concealed to principal investigators and staff members. Couples in the same household were randomly assigned as a unit. The Institutional Review Boards approved the study protocol of the recruiting centers participating in the study, and the PREDIMED-Plus trial was retrospectively registered at the International Standard Randomized Controlled Trial (ISRCTN 89898870; registration date, 24 July 2014), https://www.isrctn.com/ISRCTN8989 8870?q=ISRCTN89898870. All participants provided written informed consent.

\section{Outcome assessment}

A 17-item erMedDiet questionnaire [16] was used to assess dietary adherence to the intervention group (Additional File 1: Table s1). The 17-point scale of erMedDiet adherence is an adapted version of the previously validated 14-item questionnaire used in the PREDIMED trial [21]. This modified version includes stricter cut-off points and additional items aimed to specifically capture the potential caloric restriction for existing weight-loss goals for the erMedDiet. Compliance with each of the 17 items of the questionnaire was scored with 1 point; otherwise, the score was 0 points. As such, the erMedDiet score ranged from 0 to 17, and the higher the score, the greater the adherence. Adherence to the erMedDiet was assessed by the PREDIMED-Plus trained dietitians at baseline and at each follow-up visit.

The outcome of the present study was to attain a successful response to the dietary intervention at 6- and 12-months of follow-up. Successful dietary response was defined as an increase in at least 5 points from baseline to follow-up in the erMedDiet score or any positive increase ( $\geq 1$ point) for participants with 13 or higher scores at baseline. Participants, therefore, were classified as adherent and non-adherent based on their 17-item erMedDiet score change from baseline to 6-month and from baseline to 12-month follow-up visits (Additional File 1: Fig. s1).

\section{Covariate assessment}

Usual diet was ascertained at baseline and follow-up visits by trained dietitians throughout face-to-face interviews using the Spanish version of a previously validated 143-item semiquantitative food-frequency questionnaire (SFFQ) [22, 23]. Food consumption frequencies were registered in nine categories ranging from "never or seldom" to " $\geq 6$ times/ day" and food composition tables were used to calculate energy and nutrient intakes for each participant [24, 25]. An additional questionnaire was used to collect updated information in each visit about socio-demographics, personal and family history of disease, and lifestyles, including leisuretime physical activity, assessed by the Rapid Assessment of Physical Activity Questionnaires (RAPA-1 and RAPA2) [26], the validated Minnesota-REGICOR short physical activity questionnaire [27], and the validated Spanish version of the Nurses' Health Study questionnaire [28]. Weight and height were measured by registered dietitians with standardized procedures. Blood pressure was measured in triplicate by registered nurses using a validated semiautomatic oscillometer (Omron HEM 297 705C). Blood samples were collected after an overnight fast to determine levels of fasting blood glucose, among other determinations, with standard enzymatic methods.

\section{Independent factors}

Potential baseline factors of compliance to MedDiet were selected considering existing literature and preceding results of the PREDIMED trial [9, 10]. We categorized candidate factors in the following groups: socio-demographics, health-related characteristics, study design features, lifestyle behaviors, and baseline energy and nutrient intake. Sociodemographic characteristics included sex, age $(<65$ years, and $\geq 65$ years), marital status (married, single, widowed, and others/missing), highest attained educational level (college/university, secondary, primary or less), occupation (retired, working, unemployed/unable to work, housewife), and number of people living in the household (continuous). Health-related characteristics comprised family history of premature CVD (dichotomous), number of chronic conditions ( $\leq 3$ and $>3$ ), self-reported score of nervousness and/ or aggressiveness behavior (quartiles), body weight (continuous, per $5 \mathrm{~kg}$ ), waist circumference (continuous, per $5 \mathrm{~cm}$ ), systolic and diastolic blood pressure (both continuous, per $5 \mathrm{~mm} \mathrm{Hg}$ ), and fasting blood glucose (continuous, per $10 \mathrm{mg} / \mathrm{dL}$ ). Family history of premature CVD was defined as any immediate family member deceased by CVD 
younger than 55 years for men, and 65 years for women; for number of chronic conditions the following diagnoses were considered: hypertension, obesity (BMI $\geq 30 \mathrm{~kg} / \mathrm{m}^{2}$ ), type 2 diabetes, hypercholesterolemia, cancer, and depression; self-reported measures of nervousness and/or aggressiveness behavior were self-reported on a scale from 1 (very low selfperception) to 10 (very high self-perception). Study design features included recruitment period $(<1$ st year, between 1 st and 2 nd year, between 2 nd and 3rd year, and after $3 \mathrm{rd}$ year of recruitment), and total field center workload (below and above the median). Recruitment year was referred to the period (years) in which participants were recruited, from the date of the first recruited participant (Sep/05/2013) to the date of the last recruited participant (Oct/31/2016); total field center workload was quantified as the number of participants per center. Lifestyle behavior included leisure-time physical activity (METs-min/week, quartiles) and RAPA test (sedentary/under-active, under-active regular-activities, under-active regular, and active), 30 -s chair test (number of repeats, quartiles), smoking status (never, current, former), alcohol intake other than wine ( $\leq 5 \mathrm{~g} /$ day and $>5 \mathrm{~g} /$ day $)$, sleeping (hours, quartiles), and self-efficacy for diet modification (little/some and high). Self-efficacy for diet modification was defined as participants' beliefs in their ability to achieve dietary change with three options (little, some, or high); given the scarce number of participants responding "little", we merged the two lower categories. Energy and nutrient intake factors comprised baseline total energy intake (kcal/day, sex-specific quartiles), predefined limits of energy intake (within limits: 500-3500 kcal for women and $800-4000$ for men, and beyond limits: $<500$ or $>3500 \mathrm{kcal} /$ day for women and $<800$ or $>4000 \mathrm{kcal} /$ day for men) were used to select the analytical sample [29], fruit + vegetable consumption (g/day, sex-specific quartiles), meat consumption (g/day, sex-specific quartiles), baseline dietary fat intake (\% $\mathrm{E}$, quartiles), fiber intake (g/day, sex-specific quartiles), carbohydrate quality index (CQI) (score, quartiles), and baseline 17-point erMedDiet adherence (score, quartiles). CQI referred to the quality of dietary carbohydrate intake and was constructed upon the following four carbohydrate quality domains: high total dietary fiber intake, low glycemic index, high whole-grain carbohydrate: total grain carbohydrate ratio, and high solid carbohydrate: total carbohydrate ratio [30].

\section{Statistical analysis}

Descriptive statistics, including means and standard deviations (SD) for quantitative variables and percentages for categorical variables, were used to describe baseline characteristics of participants categorized as non-adherent and adherent according to their 17-item erMedDiet score change from baseline to 6 and 12 months of follow-up.
Chi-squared tests for categorical variables and Student's $t$ test (the assumption of normality was not violated given the large sample size) for continuous variables were used to assess differences between groups. We performed crude and adjusted multivariable logistic regression models to evaluate the probability of appropriate compliance according to the aforementioned baseline factors. Hence, odds ratios (OR) $<1$ suggest poor compliance of dietary change, whereas ORs $>1$ suggest successful compliance. For categorical variables, we used as reference the category which was expected a priori to show a greater odds of compliance, while the reference category for ordinal factors was considered the lowest category (usually, the first quartile). Tests of linear trend across categories of potential factors were run assigning the median to each category and treating the resulting variables as continuous. Participants with missing values in candidate factors were categorized as a separate group.

We conducted several sensitivity analyses using multivariable logistic regression models to corroborate the consistency of factors under different scenarios: excluding participants with any missing value; excluding participants with baseline score $\geq 13$ points in the 17 -item erMedDiet adherence questionnaire; and using an alternative definition of the outcome, especifically, scoring $>12$ points at follow-up (instead of our original definition, Additional File 1: Fig. s1).

All analyses were performed using Stata software, version 16.0 (StataCorp LP) using the PREDIMED-Plus database updated in March 2019. A two-sided $p$ value $<0.05$ was deemed as statistically significant.

\section{Results}

\section{Sample characteristics}

We excluded participants in the control group of the trial. Among the 3406 participants of the intervention arm of the PREDIMED-Plus trial, we excluded 409 (12\%) individuals with missing data on the 17-item erMedDiet questionnaire either at baseline or during follow-up, and $12(0.4 \%)$ participants with missing information of energy intake. The remaining 2985 individuals (1445 females and 1540 males) were included in our analyses. There were neither withdrawals nor losses to follow-up before the study completion (Fig. 1).

The mean (SD) age of the 2985 participants included in the study was 64.9 (4.9) years, $76 \%$ of them were married, $56 \%$ retired, $47 \%$ received primary education or less, and $45 \%$ never smoked. Baseline characteristics of participants according to their changes in the adherence to the 17 -item erMedDiet from baseline to 6-month and 12-month followup period are shown in Table 1. At 6 months, nearly half of 


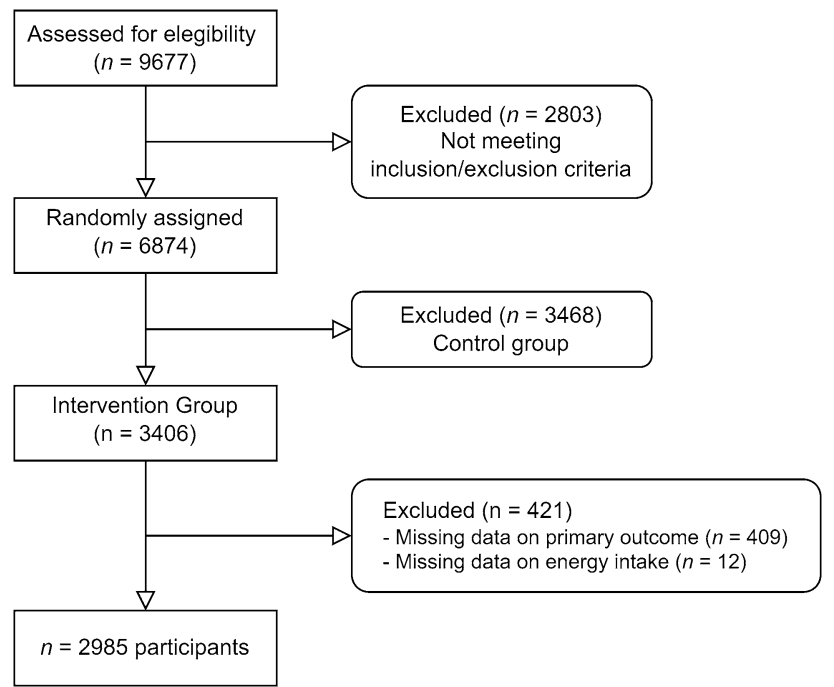

Fig. 1 Flow chart of the participants of the study. The PREDIMEDPlus trial

the participants $(49.5 \%)$ successfully attained an adequate change, while the proportion of adherent participants at 12 months was slightly higher (52.4\%).

\section{Factors of dietary change (6 months)}

Table 2 shows the main results for the crude and multivariable logistic regression analyses for the association between baseline characteristics and dietary compliance after 6 and 12 months of follow-up in the intervention group of the trial. Baseline characteristics significantly associated with better compliance at 6-month follow-up in multivariable analyses were: moderate level of physical activity (METs-min/week), high self-reported self-efficacy at baseline to change their diet, moderate consumption of fruit + vegetables, moderate meat consumption, and high fiber intake. On the other hand, being single (vs. married), having more than three chronic conditions, and being a current smoker (vs. never smoker) were associated with poorer compliance. Regarding study design features, high total field center workload was the only predictor associated with poorer compliance. Additionally, participants with a previous diagnosis of type 2 diabetes and depression were less likely to adhere to the intervention (Table 3). Noteworthy, when introducing in the model the predictor chronic conditions categorized by the number of conditions $(\leq 1,2,3,4$, and $\geq 5)$ instead of as a dichotomous variable ( $\leq 3$ and $>3$ conditions), the odds of compliance monotonically decreased as the number of chronic conditions increased (OR: $0.77,95 \%$ CI $0.56,1,04$ for 2 conditions; OR: $0.75,95 \%$ CI $0.55,1,01$ for 3 conditions; OR: $0.52,95 \%$ CI $0.37,0.73$ for 4 conditions; OR: 0.41 ,
95\% CI $0.25,0,68$ for $\geq 5$ conditions; ref.: $\leq 1$ conditions; data not shown). Additionally, the likelihood of successful compliance decreased across successive quartiles of higher baseline adherence to the erMedDiet score, probably representing a ceiling effect (Additional File 1: Table s2). The factors independently associated with dietary compliance at 6-month follow-up are shown in Fig. 2.

\section{Factors of dietary change (12 months)}

The factors associated with compliance after 12 months of follow-up identified in the logistic regression models are displayed in Table 2. Greater levels of nervousness and/or aggressiveness, regular and active levels of physical activity, high self-reported self-efficacy to modify diet, and elevated fiber intake at baseline were associated with better compliance. In turn, older age ( $\geq 65$ years), being working (vs. retired), unemployed or unable to work (vs. retired), having more than three chronic conditions, and being a current smoker (vs. never smoker) were associated with poorer compliance. For study design features, the odds of attaining compliance were significantly higher for participants recruited in the second and third years of the trial and participants belonging to field centers with a lower workload. Consistently with the results at 6-month follow-up, participants with depression less likely to comply with the intervention (Table 3). As expected because of a potential ceiling effect, participants with a higher baseline adherence to the erMedDiet showed a smaller dietary change (Additional File 1: Table s2). The factors independently associated with dietary compliance at 12-month follow-up are shown in Fig. 2.

\section{Sensitivity analyses}

We re-ran the models under different assumptions (Additional File 1: Tables s3, s4, and s5). The baseline potential factors which were consistently independently associated with successful compliance after 6 and 12 months of follow-up in all sensitivity analyses included high self-reported self-efficacy for diet modification at baseline and higher fiber intake. Consistent potential factors of poor compliance included the presence of chronic conditions (both dichotomized and categorized), depression, and higher baseline adherence to erMedDiet scale. High field center workload was the only design feature associated with poor adherence. This finding might be related to a suboptimal proportion of staff with respect to participants in the centers with a higher number of participants. Of note, the association between higher fiber intake and higher compliance became inverse when we no longer adjusted for baseline adherence 
Table 1 Baseline characteristics of the intervention group in the PREDIMED-Plus trial $(n=2,985)$ according to attained 6-month and 12-month adherence to a 17-item energy-reduced MedDiet score

\begin{tabular}{|c|c|c|c|c|c|c|}
\hline \multirow[t]{2}{*}{ Baseline characteristics } & \multicolumn{3}{|l|}{ 6-month follow-up } & \multicolumn{3}{|l|}{ 12-month follow-up } \\
\hline & $\begin{array}{l}\text { Non-adherent }{ }^{1} \\
\text { (decreasing, equal or } \\
\text { increment }<5 \text { points) } \\
(n=1507)\end{array}$ & $\begin{array}{l}\text { Adherent }{ }^{1} \text { (increas- } \\
\text { ing } \geq 5 \text { points if } \\
\text { baseline }<13 p \text { or any } \\
\text { increase if base- } \\
\text { line } \geq 13 p) \\
(n=1478)\end{array}$ & $p$ value & $\begin{array}{l}\text { Non-adherent }{ }^{1} \\
\text { (decreasing, equal or } \\
\text { increment }<5 \text { points) } \\
(n=1422)\end{array}$ & $\begin{array}{l}\text { Adherent }^{1} \text { (increas- } \\
\text { ing } \geq 5 \text { points if } \\
\text { baseline }<13 p \text { or any } \\
\text { increase if base- } \\
\text { line } \geq 13 p) \\
(n=1563)\end{array}$ & $p$ value \\
\hline \multicolumn{7}{|l|}{ Socio-demographics } \\
\hline Sex, women & 50.5 & 46.3 & 0.021 & 51.1 & 45.9 & 0.005 \\
\hline Age, years, mean (SD) & $65.2(4.9)$ & $64.7(5.0)$ & 0.005 & $65.2(5.0)$ & $64.7(4.9)$ & 0.016 \\
\hline Marital status & & & 0.034 & & & 0.020 \\
\hline Married & 73.8 & 77.2 & & 73.1 & 77.6 & \\
\hline Single & 6.4 & 4.3 & & 6.0 & 4.8 & \\
\hline Widowed & 10.9 & 10.9 & & 11.3 & 10.5 & \\
\hline Others/misisng & 9.0 & 7.6 & & 9.6 & 7.1 & \\
\hline $\begin{array}{l}\text { Attained education } \\
\text { level }\end{array}$ & & & 0.621 & & & 0.725 \\
\hline College/university & 22.2 & 20.8 & & 22.1 & 21.0 & \\
\hline Secondary & 30.7 & 30.5 & & 31.1 & 30.1 & \\
\hline Primary or less & 46.4 & 47.5 & & 45.9 & 47.9 & \\
\hline Occupation & & & 0.657 & & & 0.833 \\
\hline Retired & 55.9 & 55.6 & & 55.0 & 56.5 & \\
\hline Working & 20.1 & 22.0 & & 21.0 & 21.1 & \\
\hline $\begin{array}{l}\text { Unemployed or } \\
\text { unable to work }\end{array}$ & 8.0 & 7.9 & & 8.2 & 7.7 & \\
\hline Housewife & 15.3 & 13.9 & & 15.1 & 14.1 & \\
\hline $\begin{array}{l}\text { Number of people in } \\
\text { household, mean } \\
\text { (SD) }\end{array}$ & $1.4(1.1)$ & $1.4(1.2)$ & 0.351 & $1.4(1.1)$ & $1.4(1.0)$ & 0.069 \\
\hline \multicolumn{7}{|l|}{$\begin{array}{l}\text { Baseline health-related } \\
\text { characteristics }\end{array}$} \\
\hline Hypertension & 84.4 & 82.9 & 0.260 & 83.5 & 83.8 & 0.802 \\
\hline Obesity & 72.7 & 72.7 & 0.965 & 71.9 & 73.4 & 0.334 \\
\hline Type 2 diabetes & 32.1 & 23.5 & $<0.001$ & 31.3 & 24.7 & $<0.001$ \\
\hline Hypercholesterolemia & 71.1 & 68.7 & 0.154 & 70.1 & 69.8 & 0.853 \\
\hline $\begin{array}{l}{ }^{2} \text { Family history of } \\
\text { premature CVD }\end{array}$ & 12.3 & 13.1 & 0.557 & 12.9 & 12.5 & 0.787 \\
\hline Cancer & 7.6 & 7.1 & 0.582 & 7.5 & 7.3 & 0.867 \\
\hline Depression & 21.0 & 17.6 & 0.017 & 21.9 & 17.0 & 0.001 \\
\hline $\begin{array}{l}{ }^{3} \text { Average number of } \\
\text { chronic conditions, } \\
\text { mean (SD) }\end{array}$ & $2.9(1.0)$ & $2.7(1.0)$ & $<0.001$ & $2.9(1.0)$ & $2.8(1.0)$ & 0.007 \\
\hline $\begin{array}{l}{ }^{4} \text { Self-reported meas- } \\
\text { ure of nervousness } \\
\text { and/or aggressive- } \\
\text { ness behavior }\end{array}$ & & & 0.717 & & & 0.219 \\
\hline Q1 (low) & 27.3 & 27.4 & & 28.4 & 26.4 & \\
\hline Q2 & 37.6 & 35.8 & & 37.5 & 36.0 & \\
\hline Q3 & 11.5 & 11.8 & & 11.3 & 11.9 & \\
\hline Q4 (high) & 23.6 & 25.0 & & 22.8 & 25.7 & \\
\hline $\begin{array}{l}\text { Body weight, kg, } \\
\text { mean (SD) }\end{array}$ & $85.8(12.9)$ & $87.2(13.1)$ & 0.003 & $85.9(13.3)$ & $87.0(12.8)$ & 0.023 \\
\hline
\end{tabular}


Table 1 (continued)

\begin{tabular}{|c|c|c|c|c|c|c|}
\hline \multirow[t]{2}{*}{ Baseline characteristics } & \multicolumn{3}{|l|}{ 6-month follow-up } & \multicolumn{3}{|l|}{ 12-month follow-up } \\
\hline & $\begin{array}{l}\text { Non-adherent }{ }^{1} \\
\text { (decreasing, equal or } \\
\text { increment }<5 \text { points) } \\
(n=1507)\end{array}$ & $\begin{array}{l}\text { Adherent }{ }^{1} \text { (increas- } \\
\text { ing } \geq 5 \text { points if } \\
\text { baseline }<13 p \text { or any } \\
\text { increase if base- } \\
\text { line } \geq 13 p) \\
(n=1478)\end{array}$ & $p$ value & $\begin{array}{l}\text { Non-adherent }{ }^{1} \\
\text { (decreasing, equal or } \\
\text { increment }<5 \text { points) } \\
(n=1422)\end{array}$ & $\begin{array}{l}\text { Adherent }{ }^{1} \text { (increas- } \\
\text { ing } \geq 5 \text { points if } \\
\text { baseline }<13 p \text { or any } \\
\text { increase if base- } \\
\text { line } \geq 13 p) \\
(n=1563)\end{array}$ & $p$ value \\
\hline $\begin{array}{l}\text { Waist circumference, } \\
\mathrm{cm} \text {, mean }(\mathrm{SD})\end{array}$ & $107(10)$ & $108(10)$ & 0.013 & $107(10)$ & $108(10)$ & 0.213 \\
\hline $\begin{array}{l}\text { BMI, } \mathrm{kg} / \mathrm{m}^{2}, \text { mean } \\
\text { (SD) }\end{array}$ & $32.5(3.4)$ & $32.6(3.5)$ & 0.299 & $32.5(3.5)$ & $32.6(3.4)$ & 0.541 \\
\hline $\begin{array}{l}\text { SBP, mmHg, mean } \\
\text { (SD) }\end{array}$ & $139(18)$ & $140(17)$ & 0.014 & $139(17)$ & $140(17)$ & 0.191 \\
\hline $\begin{array}{l}\text { DBP, mmHg, mean } \\
\text { (SD) }\end{array}$ & 80.5 (9.9) & $81.1(9.9)$ & 0.096 & $80.7(10.2)$ & $81(9.7)$ & 0.464 \\
\hline $\begin{array}{l}\text { Fasting blood glucose, } \\
\text { mg/dL, mean (SD) }\end{array}$ & $113(28)$ & $112(26)$ & 0.197 & $113(28)$ & $112(27)$ & 0.249 \\
\hline \multicolumn{7}{|l|}{ Study design features } \\
\hline${ }^{5}$ Recruitment year & & & 0.005 & & & $<0.001$ \\
\hline$<1$ st & 10.6 & 7.4 & & 11.3 & 6.9 & \\
\hline 1st-2nd & 25.4 & 25.6 & & 24.0 & 26.9 & \\
\hline $2 \mathrm{nd}-3 \mathrm{rd}$ & 48.0 & 52.9 & & 49.0 & 51.8 & \\
\hline$>3 \mathrm{rd}$ & 16.1 & 14.1 & & 15.8 & 14.4 & \\
\hline $\begin{array}{l}{ }^{6} \text { Total workload of } \\
\text { center, participants, } \\
\text { mean (SD) }\end{array}$ & $151(64)$ & $154(62)$ & 0.330 & $152(64)$ & $153(62)$ & 0.547 \\
\hline \multicolumn{7}{|l|}{$\begin{array}{l}\text { Baseline lifestyles and } \\
\text { behaviors }\end{array}$} \\
\hline \multicolumn{7}{|l|}{ Physical activity } \\
\hline $\begin{array}{l}\text { METs-min/week, } \\
\text { mean }(\mathrm{SD})\end{array}$ & 2498 (2312) & $2332(2170)$ & 0.043 & $2398(2180)$ & $2431(2301)$ & 0.687 \\
\hline RAPA test & & & 0.278 & & & 0.197 \\
\hline $\begin{array}{l}\text { Level } 1 \text { (sedentary } \\
\text { or under-active) }\end{array}$ & 18.4 & 18.8 & & 19.5 & 17.9 & \\
\hline $\begin{array}{l}\text { Level } 2 \text { (under- } \\
\text { active regular- } \\
\text { light activities) }\end{array}$ & 35.3 & 37.0 & & 36.8 & 35.6 & \\
\hline $\begin{array}{l}\text { Level } 3 \text { (under- } \\
\text { active regular) }\end{array}$ & 17.3 & 18.3 & & 16.3 & 19.1 & \\
\hline Level 4 (active) & 29.0 & 25.8 & & 27.4 & 27.4 & \\
\hline $\begin{array}{l}\text { Chair test } 30 \mathrm{~s} \text {, } \\
\text { repeats, mean }(\mathrm{SD})\end{array}$ & $13.1(4.9)$ & $13.4(4.7)$ & 0.140 & $13.1(4.9)$ & $13.4(4.7)$ & 0.053 \\
\hline Smoking status, n (\%) & & & 0.574 & & & 0.469 \\
\hline Never smokers & 44.3 & 45.3 & & 44.4 & 45.1 & \\
\hline Current smokers & 13.9 & 12.7 & & 14.2 & 12.5 & \\
\hline Former smokers & 41.2 & 41.7 & & 40.8 & 42.0 & \\
\hline $\begin{array}{l}\text { Alcohol intake other } \\
\text { than wine, g/day, } \\
\text { mean (SD) }\end{array}$ & $4.5(8.6)$ & $4.7(8.3)$ & 0.272 & $4.5(9.0)$ & $4.6(8.0)$ & 0.210 \\
\hline $\begin{array}{l}\text { Sleeping, hours/day, } \\
\text { mean (SD) }\end{array}$ & $7.0(1.2)$ & $7.0(1.2)$ & 0.644 & $7.0(1.2)$ & $7.0(1.2)$ & 0.960 \\
\hline $\begin{array}{l}\text { High perceived self- } \\
\text { efficacy for diet } \\
\text { modification }\end{array}$ & 72.9 & 77.7 & 0.003 & 71.9 & 78.3 & $<0.001$ \\
\hline
\end{tabular}


Table 1 (continued)

\begin{tabular}{|c|c|c|c|c|c|c|}
\hline \multirow[t]{2}{*}{ Baseline characteristics } & \multicolumn{3}{|l|}{ 6-month follow-up } & \multicolumn{3}{|l|}{ 12-month follow-up } \\
\hline & $\begin{array}{l}\text { Non-adherent }{ }^{1} \\
\text { (decreasing, equal or } \\
\text { increment }<5 \text { points) } \\
(n=1507)\end{array}$ & $\begin{array}{l}\text { Adherent }{ }^{1} \text { (increas- } \\
\text { ing } \geq 5 \text { points if } \\
\text { baseline }<13 p \text { or any } \\
\text { increase if base- } \\
\text { line } \geq 13 p) \\
(n=1478)\end{array}$ & $p$ value & $\begin{array}{l}\text { Non-adherent }{ }^{1} \\
\text { (decreasing, equal or } \\
\text { increment }<5 \text { points) } \\
(n=1422)\end{array}$ & $\begin{array}{l}\text { Adherent }{ }^{1} \text { (increas- } \\
\text { ing } \geq 5 \text { points if } \\
\text { baseline }<13 p \text { or any } \\
\text { increase if base- } \\
\text { line } \geq 13 p) \\
(n=1563)\end{array}$ & $p$ value \\
\hline \multicolumn{7}{|l|}{$\begin{array}{l}\text { Baseline dietary pat- } \\
\text { tern, total energy, and } \\
\text { nutrient intake }\end{array}$} \\
\hline $\begin{array}{l}\text { Baseline } 17 \text {-item } \\
\text { energy-reduced } \\
\text { MedDiet score, } \\
\text { mean (SD) }\end{array}$ & $9.4(2.3)$ & $7.5(2.6)$ & $<0.001$ & $9.5(2.3)$ & $7.5(2.6)$ & $<0.001$ \\
\hline $\begin{array}{l}\text { Total energy intake, } \\
\text { (kcal/day), mean } \\
\text { (SD) }\end{array}$ & $2365(610)$ & $2438(588)$ & 0.001 & $2356(599)$ & $2443(598)$ & $<0.001$ \\
\hline $\begin{array}{l}\text { Participants with } \\
\text { total energy intake } \\
\text { beyond predefined } \\
\text { limits (Willett) }\end{array}$ & 2.5 & 2.4 & 0.877 & 2.2 & 2.6 & 0.431 \\
\hline $\begin{array}{l}\text { Baseline fruit + veg- } \\
\text { etable consumption, } \\
\text { g/day, mean (SD) }\end{array}$ & $714(293)$ & $676(266)$ & $<0.001$ & $716(290)$ & $677(270)$ & $<0.001$ \\
\hline $\begin{array}{l}\text { Baseline meat con- } \\
\text { sumption, g/day, } \\
\text { mean (SD) }\end{array}$ & $146(60)$ & $152(59)$ & 0.003 & $146(60)$ & $151(59)$ & 0.008 \\
\hline $\begin{array}{l}\text { Baseline dietary fat } \\
\text { intake, \% E, mean } \\
\text { (SD) }\end{array}$ & $39.4(6.8)$ & $39.6(6.3)$ & 0.352 & $39.7(6.7)$ & $39.4(6.4)$ & 0.251 \\
\hline $\begin{array}{l}\text { Baseline fiber intake, } \\
\text { g/day, mean (SD) }\end{array}$ & $27.2(9.4)$ & $26.1(8.7)$ & $<0.001$ & $27.2(9.5)$ & $26.1(8.7)$ & $<0.001$ \\
\hline $\begin{array}{l}\text { Baseline carbohydrate } \\
\text { Quality Index }{ }^{7}, \\
\text { mean (SD) }\end{array}$ & $10.1(2.6)$ & $9.6(2.6)$ & $<0.001$ & $10.2(2.5)$ & $9.6(2.6)$ & $<0.001$ \\
\hline
\end{tabular}

Values are percentages of participants unless otherwise indicated.

$B M I$ body mass index, $C C$ chronic conditions, $C V D$ cardiovascular disease, $D B P$ diastolic blood pressure, $E$ energy, MedDiet Mediterranean diet, $M E T$ metabolic equivalent, $Q$ quartile, $R A P A$ rapid assessment of physical activity, $S B P$ systolic blood pressure

Data available in the intervention group of the PREDIMED-Plus trial $(n=2985)$; for marital status $(n=10$ missing); for attained education level ( $n=29$ missing); for occupation ( $n=20$ missing); for number of people in household ( $n=5$ missing); for self-reported measure of nervousness and/or aggressiveness behavior ( $n=26$ missing); for SBP ( $n=23$ missing); for DBP ( $n=23$ missing); for fasting blood glucose ( $n=42 \mathrm{missing}$ ); for RAPA test ( $n=1$ missing); for smoking status ( $n=14$ missing); for sleeping hours ( $n=38$ missing).

${ }^{1}$ Adherence to Mediterranean diet was evaluated using a 17-point scale of adherence to an energy-reduced MedDiet questionnaire (1 point for each item). Participants with an increase of $\geq 5$ points from baseline to follow-up were classified in the "adherent group". Participants with $\geq 13$ points at baseline and any positive increase ( $\geq 1$ point) from baseline to follow-up were additionally classified in the "adherent group". Detailed information is provided in Additional File 1: Figure s1.

${ }^{2}$ Family history of premature CVD was defined as any immediate family member deceased younger than 55 years for men and 65 years for women.

${ }^{3}$ Number of chronic conditions was calculated by summing the following chronic conditions (1 point for each condition): hypertension, obesity, type 2 diabetes, hypercholesterolemia, cancer, and depression).

${ }^{4}$ Self-reported measure of nervousness and/or aggressiveness behavior was reported on a scale from 1 (very low self-perception) to 10 (very high self-perception).

${ }^{5}$ Recruitment year was referred to the period (years) in which participants were recruited, from the date of the first recruited participant to the date of the last recruited participant $(<1,1-2,2-3$, and $>3$ years $)$.

${ }^{6}$ Total workload of center was measured as the number of participants in the intervention group per center

${ }^{7}$ Carbohydrate Quality Index was referred to the quality of dietary carbohydrate intake (high total dietary fiber intake, low glycemic index, high whole-grain carbohydrate: total grain carbohydrate ratio, and high solid carbohydrate: total carbohydrate ratio. 
Table 2 Odds ratios (OR) and 95\% confidence intervals (95\% CI) of attaining good adherence ${ }^{1}$ (increasing $\geq 5$ points if baseline $<13 p$ or any increase if baseline $\geq 13 p$ ) to the MedDiet intervention at 6 and
12 months of follow-up in the active intervention group of the PREDIMED-Plus trial $(n=2,985)$

\begin{tabular}{|c|c|c|c|c|c|c|c|c|c|}
\hline \multirow[t]{3}{*}{ Baseline characteristics } & \multirow[t]{3}{*}{$n$} & \multicolumn{8}{|c|}{$\begin{array}{l}\text { OR }(95 \% \mathrm{CI}) \text { for adherence (increasing } \geq 5 \text { points if baseline }<13 \mathrm{p} \text { or any increase if baseline } \geq 13 \mathrm{p})^{1} \text { to the } \\
\text { MedDiet intervention (adherent vs. non-adherent) }{ }^{2}\end{array}$} \\
\hline & & \multicolumn{4}{|l|}{6 month-follow-up } & \multicolumn{4}{|c|}{12 month-follow-up } \\
\hline & & Crude $^{3}$ & $p$ value & Multivariable $^{4}$ & $p$ value & Crude $^{3}$ & $p$ value & Multivariable $^{4}$ & $p$ value \\
\hline \multicolumn{10}{|l|}{ Socio-demographics } \\
\hline \multicolumn{10}{|l|}{ Sex } \\
\hline Men & 1540 & 1.00 (ref) & - & 1.00 (ref) & - & 1.00 (ref) & - & 1.00 (ref) & - \\
\hline Women & 1445 & $0.84(0.73-0.97)$ & 0.021 & $1.19(0.93-1.52)$ & 0.173 & $0.81(0.70-0.94)$ & 0.005 & $1.10(0.86-1.40)$ & 0.470 \\
\hline \multicolumn{10}{|l|}{ Age, years } \\
\hline$<65$ & 1404 & 1.00 (ref) & - & 1.00 (ref) & - & 1.00 (ref) & - & 1.00 (ref) & - \\
\hline$\geq 65$ & 1581 & $0.88(0.77-1.02)$ & 0.094 & $0.86(0.70-1.07)$ & 0.171 & $0.89(0.77-1.03)$ & 0.109 & $0.80(0.65-0.99)$ & 0.039 \\
\hline \multicolumn{10}{|l|}{ Marital status } \\
\hline Married & 2253 & 1.00 (ref) & - & 1.00 (ref) & - & 1.00 (ref) & - & 1.00 (ref) & - \\
\hline Single & 160 & $0.65(0.47-0.90)$ & 0.010 & $0.64(0.44-0.93)$ & 0.020 & $0.76(0.55-1.04)$ & 0.089 & $0.83(0.57-1.20)$ & 0.317 \\
\hline Widowed & 325 & $0.96(0.76-1.21)$ & 0.710 & $0.97(0.73-1.29)$ & 0.837 & $0.87(0.69-1.10)$ & 0.254 & $0.92(0.69-1.22)$ & 0.544 \\
\hline Others/missing & 247 & $0.81(0.62-1.05)$ & 0.114 & $0.84(0.62-1.14)$ & 0.253 & $0.70(0.54-0.91)$ & 0.008 & $0.75(0.55-1.02)$ & 0.066 \\
\hline \multicolumn{10}{|l|}{ Attained education level } \\
\hline College/university & 642 & 1.00 (ref) & - & 1.00 (ref) & & 1.00 (ref) & - & 1.00 (ref) & - \\
\hline Secondary & 913 & $1.06(0.86-1.30)$ & 0.581 & $0.94(0.75-1.18)$ & 0.602 & $1.02(0.83-1.25)$ & 0.847 & $0.85(0.67-1.07)$ & 0.164 \\
\hline Primary or less & 1401 & $1.09(0.90-1.31)$ & 0.371 & $1.06(0.84-1.33)$ & 0.641 & $1.10(0.91-1.33)$ & 0.319 & $1.01(0.80-1.27)$ & 0.938 \\
\hline Missing & 29 & $1.54(0.72-3.27)$ & 0.265 & $1.28(0.56-2.96)$ & 0.558 & $1.03(0.49-2.16)$ & 0.947 & $0.80(0.35-1.85)$ & 0.602 \\
\hline \multicolumn{10}{|l|}{ Occupation } \\
\hline Retired & 1665 & 1.00 (ref) & - & 1.00 (ref) & - & 1.00 (ref) & - & 1.00 (ref) & - \\
\hline Working & 628 & $1.10(0.92-1.32)$ & 0.309 & $0.88(0.68-1.14)$ & 0.321 & $0.98(0.82-1.18)$ & 0.836 & $0.72(0.56-0.93)$ & 0.013 \\
\hline $\begin{array}{l}\text { Unemployed or } \\
\text { unable to work }\end{array}$ & 237 & $1.00(0.76-1.31)$ & 0.999 & $0.81(0.58-1.14)$ & 0.226 & $0.92(0.70-1.21)$ & 0.568 & $0.69(0.49-0.96)$ & 0.029 \\
\hline Housewife & 435 & $0.91(0.74-1.13)$ & 0.405 & $0.85(0.65-1.11)$ & 0.228 & $0.91(0.73-1.12)$ & 0.361 & $0.88(0.67-1.15)$ & 0.363 \\
\hline Missing & 20 & $0.84(0.35-2.04)$ & 0.698 & $0.99(0.36-2.70)$ & 0.984 & $0.72(0.30-1.76)$ & 0.476 & $0.88(0.32-2.39)$ & 0.799 \\
\hline $\begin{array}{l}\text { Number of people in } \\
\text { household (continu- } \\
\text { ous) }\end{array}$ & 2985 & $1.03(0.96-1.11)$ & 0.344 & $0.96(0.88-1.05)$ & 0.387 & $1.07(0.99-1.14)$ & 0.070 & $1.00(0.92-1.09)$ & 0.943 \\
\hline \multicolumn{10}{|c|}{ Health-related characteristics } \\
\hline \multicolumn{10}{|c|}{${ }^{5}$ Family history of premature CVD } \\
\hline No & 2606 & 1.00 (ref) & - & 1.00 (ref) & - & 1.00 (ref) & - & 1.00 (ref) & - \\
\hline Yes & 379 & $1.07(0.86-1.32)$ & 0.557 & $1.09(0.86-1.39)$ & 0.476 & $0.97(0.78-1.20)$ & 0.787 & $0.94(0.74-1.20)$ & 0.629 \\
\hline \multicolumn{10}{|c|}{${ }^{6}$ Number of chronic conditions } \\
\hline$\leq 3$ & 2267 & 1.00 (ref) & - & 1.00 (ref) & - & 1.00 (ref) & - & 1.00 (ref) & - \\
\hline$>3$ & 718 & $0.67(0.56-0.79)$ & $<0.001$ & $0.65(0.53-0.79)$ & $<0.001$ & $0.72(0.61-0.86)$ & $<0.001$ & $0.76(0.62-0.93)$ & 0.009 \\
\hline \multicolumn{10}{|c|}{${ }^{7,8}$ Self-reported measure of nervousness and/or aggressiveness behavior, score } \\
\hline $\mathrm{Q} 1(<4)$ & 816 & 1.00 (ref) & - & 1.00 (ref) & - & 1.00 (ref) & - & 1.00 (ref) & - \\
\hline $\mathrm{Q} 2(4-5)$ & 1096 & $0.95(0.79-1.13)$ & & $0.89(0.72-1.09)$ & & $1.04(0.86-1.24)$ & & $1.01(0.82-1.24)$ & \\
\hline Q3 (6) & 347 & $1.02(0.79-1.31)$ & & $0.92(0.69-1.21)$ & & $1.13(0.88-1.46)$ & & $1.04(0.79-1.39)$ & \\
\hline Q4 (>6) & 726 & $1.05(0.86-1.29)$ & 0.691 & $1.10(0.88-1.38)$ & 0.696 & $1.22(1.00-1.49)$ & 0.066 & $1.32(1.05-1.66)$ & 0.045 \\
\hline $\begin{array}{l}\text { Body weight, (per } \\
5 \mathrm{~kg} \text { ) }\end{array}$ & 2985 & $1.04(1.01-1.07)$ & 0.003 & $1.02(0.96-1.08)$ & 0.522 & $1.03(1.01-1.06)$ & 0.023 & $1.03(0.97-1.09)$ & 0.328 \\
\hline $\begin{array}{l}\text { Waist circumference } \\
\text { (per } 5 \mathrm{~cm})\end{array}$ & 2985 & 1.05 (1.01-1.09) & 0.014 & $1.02(0.94-1.09)$ & 0.681 & $1.02(0.99-1.06)$ & 0.213 & $0.97(0.90-1.05)$ & 0.476 \\
\hline $\mathrm{SBP}$ (per $5 \mathrm{~mm} \mathrm{Hg}$ ) & 2985 & $1.03(1.01-1.05)$ & 0.013 & $1.02(1.00-1.06)$ & 0.098 & $1.01(0.99-1.04)$ & 0.190 & $1.00(0.98-1.03)$ & 0.766 \\
\hline DBP (per $5 \mathrm{~mm} \mathrm{Hg}$ ) & 2985 & $1.03(0.99-1.07)$ & 0.100 & $1.02(0.97-1.07)$ & 0.414 & $1.01(0.98-1.05)$ & 0.487 & $1.01(0.96-1.07)$ & 0.567 \\
\hline
\end{tabular}


Table 2 (continued)

Baseline characteristics $n \quad$ OR $(95 \% \mathrm{CI})$ for adherence (increasing $\geq 5$ points if baseline $<13 p$ or any increase if baseline $\geq 13 p)^{1}$ to the MedDiet intervention (adherent vs. non-adherent) ${ }^{2}$

\begin{tabular}{|c|c|c|c|c|c|c|c|c|c|}
\hline & \multirow{2}{*}{\multicolumn{4}{|c|}{6 month-follow-up }} & \multirow{2}{*}{\multicolumn{4}{|c|}{12 month-follow-up }} \\
\hline & & & & & & & & & \\
\hline & & Crude $^{3}$ & $p$ value & Multivariable $^{4}$ & $p$ value & Crude $^{3}$ & $p$ value & Multivariable $^{4}$ & $p$ value \\
\hline $\begin{array}{l}\text { Fasting blood glucose } \\
\text { (per } 10 \mathrm{mg} / \mathrm{dL} \text { ) }\end{array}$ & 2985 & $0.98(0.96-1.01)$ & 0.147 & $1.00(0.97-1.03)$ & 0.900 & $0.98(0.96-1.01)$ & 0.223 & $1.00(0.97-1.03)$ & 0.864 \\
\hline \multicolumn{10}{|l|}{ Study design features } \\
\hline \multicolumn{10}{|l|}{${ }^{9}$ Recruitment year } \\
\hline$<1$ st & 268 & 1.00 (ref) & - & 1.00 (ref) & - & 1.00 (ref) & - & 1.00 (ref) & - \\
\hline $1 \mathrm{st}-2 \mathrm{nd}$ & 761 & 1.45 (1.09-1.92) & 0.010 & $1.11(0.81-1.53)$ & 0.510 & $1.82(1.38-2.42)$ & $<0.001$ & $1.57(1.14-2.17)$ & 0.005 \\
\hline $2 \mathrm{nd}-3 \mathrm{rd}$ & 1506 & $1.57(1.20-2.04)$ & 0.001 & $1.28(0.94-1.74)$ & 0.112 & $1.73(1.33-2.25)$ & $<0.001$ & $1.63(1.20-2.21)$ & 0.002 \\
\hline$>3 \mathrm{rd}$ & 450 & $1.28(0.95-1.74)$ & 0.109 & $0.98(0.69-1.40)$ & 0.919 & $1.48(1.09-2.01)$ & 0.012 & $1.36(0.95-1.94)$ & 0.088 \\
\hline \multicolumn{10}{|c|}{${ }^{10}$ Total workload of center, participants in intervention group } \\
\hline $\begin{array}{l}\text { Below median ( } \\
n \leq 128)\end{array}$ & 1498 & 1.00 (ref) & - & 1.00 (ref) & - & 1.00 (ref) & - & 1.00 (ref) & - \\
\hline $\begin{array}{l}\text { Above median } \\
\quad(n>128)\end{array}$ & 1487 & $0.81(0.70-0.93)$ & 0.003 & $0.76(0.65-0.90)$ & 0.002 & $0.83(0.72-0.96)$ & 0.014 & $0.81(0.69-0.96)$ & 0.016 \\
\hline \multicolumn{10}{|l|}{ Lifestyle behavior } \\
\hline \multicolumn{10}{|l|}{ Physical activity } \\
\hline $\mathrm{Q} 1(<840)$ & 778 & 1.00 (ref) & - & 1.00 (ref) & - & 1.00 (ref) & - & 1.00 (ref) & - \\
\hline Q2 (840-1818) & 720 & $1.22(0.99-1.49)$ & & $1.35(1.06-1.71)$ & & $1.01(0.82-1.23)$ & & $0.99(0.78-1.26)$ & \\
\hline Q3 (1819-3356) & 762 & $1.09(0.89-1.33)$ & & $1.27(0.99-1.62)$ & & $1.01(0.82-1.23)$ & & $1.05(0.82-1.34)$ & \\
\hline $\mathrm{Q} 4(>3356)$ & 725 & $0.88(0.72-1.08)$ & 0.057 & $1.04(0.80-1.36)$ & 0.619 & $1.04(0.85-1.27)$ & 0.717 & $1.10(0.84-1.43)$ & 0.412 \\
\hline \multicolumn{10}{|l|}{ RAPA test } \\
\hline $\begin{array}{l}\text { Level } 1 \text { (sedentary } \\
\text { or under-active) }\end{array}$ & 556 & 1.00 (ref) & - & 1.00 (ref) & - & 1.00 (ref) & - & 1.00 (ref) & - \\
\hline $\begin{array}{l}\text { Level } 2 \text { (under- } \\
\text { active regular- } \\
\text { light activities) }\end{array}$ & 1079 & $1.03(0.84-1.26)$ & 0.790 & $1.01(0.80-1.28)$ & 0.919 & $1.06(0.86-1.30)$ & 0.605 & $1.07(0.84-1.35)$ & 0.579 \\
\hline $\begin{array}{l}\text { Level } 3 \text { (under- } \\
\text { active regular) }\end{array}$ & 531 & $1.04(0.82-1.32)$ & 0.733 & $1.12(0.84-1.49)$ & 0.436 & $1.28(1.01-1.62)$ & 0.043 & $1.50(1.12-2.00)$ & 0.006 \\
\hline Level 4 (active) & 819 & $0.87(0.70-1.08)$ & 0.221 & $1.13(0.86-1.50)$ & 0.378 & $1.09(0.88-1.35)$ & 0.423 & $1.46(1.10-1.94)$ & 0.008 \\
\hline \multicolumn{10}{|l|}{${ }^{8}$ Chair test $30 \mathrm{~s}$, repeats } \\
\hline $\mathrm{Q} 1(<12)$ & 962 & 1.00 (ref) & - & 1.00 (ref) & - & 1.00 (ref) & - & 1.00 (ref) & - \\
\hline Q2 (12-13) & 665 & $1.17(0.96-1.43)$ & & $1.06(0.84-1.32)$ & & $1.13(0.93-1.38)$ & & $0.99(0.79-1.24)$ & \\
\hline Q3 (14-16) & 762 & $1.07(0.89-1.30)$ & & $1.01(0.81-1.26)$ & & $1.14(0.94-1.38)$ & & $1.04(0.83-1.30)$ & \\
\hline $\mathrm{Q} 4(>16)$ & 596 & $1.10(0.89-1.34)$ & 0.552 & $0.94(0.73-1.20)$ & 0.563 & $1.11(0.91-1.37)$ & 0.301 & $0.89(0.69-1.14)$ & 0.440 \\
\hline \multicolumn{10}{|l|}{ Smoking status, $n(\%)$} \\
\hline Never smokers & 1337 & 1.00 (ref) & - & 1.00 (ref) & - & 1.00 (ref) & - & 1.00 (ref) & - \\
\hline Current smokers & 397 & $0.90(0.72-1.12)$ & 0.348 & $0.75(0.57-0.98)$ & 0.038 & $0.87(0.69-1.08)$ & 0.206 & $0.68(0.52-0.89)$ & 0.005 \\
\hline Former smokers & 1237 & $0.99(0.85-1.16)$ & 0.903 & $0.95(0.78-1.16)$ & 0.634 & $1.02(0.87-1.19)$ & 0.846 & $0.94(0.77-1.14)$ & 0.533 \\
\hline Missing & 14 & $0.55(0.18-1.66)$ & 0.293 & $0.42(0.13-1.40)$ & 0.159 & $0.67(0.23-1.95)$ & 0.465 & $0.58(0.18-1.88)$ & 0.361 \\
\hline \multicolumn{10}{|c|}{ Alcohol intake other than wine } \\
\hline Never & 997 & 1.00 (ref) & - & 1.00 (ref) & - & 1.00 (ref) & - & 1.00 (ref) & - \\
\hline$\leq 5 \mathrm{~g} /$ day & 1266 & $1.05(0.89-1.23)$ & 0.603 & $0.93(0.76-1.12)$ & 0.432 & $1.07(0.91-1.27)$ & 0.411 & $0.95(0.78-1.16)$ & 0.619 \\
\hline$>5$ g/day & 722 & $1.12(0.93-1.36)$ & 0.235 & $0.95(0.74-1.22)$ & 0.683 & $1.19(0.99-1.45)$ & 0.069 & $1.00(0.78-1.28)$ & 0.993 \\
\hline \multicolumn{10}{|l|}{${ }^{8}$ Sleeping, hours/day } \\
\hline $\mathrm{Q} 1(<7)$ & 964 & 1.00 (ref) & - & 1.00 (ref) & - & 1.00 (ref) & - & 1.00 (ref) & - \\
\hline Q2 (7) & 984 & $0.99(0.83-1.18)$ & & $0.98(0.80-1.20)$ & & $0.94(0.79-1.12)$ & & $0.91(0.75-1.12)$ & \\
\hline Q3 (8) & 777 & $0.87(0.72-.1 .05)$ & & $0.86(0.70-1.06)$ & & $0.99(0.82-1.20)$ & & $1.00(0.81-1.24)$ & \\
\hline Q4 (>8) & 260 & $1.14(0.87-1.50)$ & 0.763 & $1.10(0.80-1.50)$ & 0.634 & $1.11(0.85-1.47)$ & 0.602 & $1.00(0.74-1.37)$ & 0.910 \\
\hline
\end{tabular}


Table 2 (continued)

Baseline characteristics $n \quad$ OR $(95 \% \mathrm{CI})$ for adherence (increasing $\geq 5$ points if baseline $<13 p$ or any increase if baseline $\geq 13 p)^{1}$ to the MedDiet intervention (adherent vs. non-adherent) ${ }^{2}$

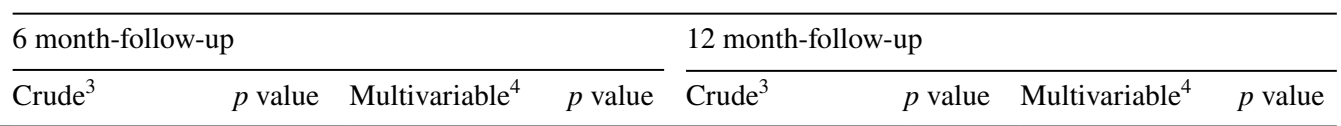

Self-efficacy for diet modification

\begin{tabular}{|c|c|c|c|c|c|c|c|c|c|}
\hline Little or some & 738 & 1.00 (ref) & - & 1.00 (ref) & - & 1.00 (ref) & - & 1.00 (ref) & - \\
\hline High & 2247 & $1.29(1.09-1.53)$ & 0.003 & $1.51(1.25-1.83)$ & $<0.001$ & $1.41(1.19-1.66)$ & $<0.001$ & $1.66(1.37-2.01)$ & $<0.001$ \\
\hline
\end{tabular}

Total energy and nutrient intake

${ }^{8}$ Total energy intake, kcal/day

\begin{tabular}{|c|c|c|c|c|c|c|c|c|c|}
\hline $\begin{array}{r}\text { Q1 }(\text { men }<2121 \text {; } \\
\text { women < 1889) }\end{array}$ & 747 & 1.00 (ref) & - & 1.00 (ref) & - & 1.00 (ref) & - & 1.00 (ref) & - \\
\hline $\begin{array}{l}\text { Q2 (men 2121- } \\
\text { 2477; women: } \\
\text { 1889-2214) }\end{array}$ & 746 & $1.20(0.98-1.47)$ & & $1.00(0.78-1.27)$ & & $1.15(0.94-1.40)$ & & $0.99(0.78-1.27)$ & \\
\hline $\begin{array}{l}\text { Q3 (men 2478- } \\
\text { 2885; women: } \\
\text { 2215-2564) }\end{array}$ & 746 & $1.41(1.15-1.73)$ & & $1.02(0.79-1.32)$ & & $1.43(1.17-1.75)$ & & $1.10(0.84-1.42)$ & \\
\hline $\begin{array}{l}\text { Q4 }(\text { men }>2885 \\
\text { women: }>2564)\end{array}$ & 746 & $1.36(1.11-1.67)$ & 0.002 & $0.84(0.62-1.13)$ & 0.234 & $1.35(1.11-1.66)$ & 0.001 & $0.88(0.66-1.19)$ & 0.464 \\
\hline \multicolumn{10}{|c|}{ redefined limits of energy intake (Willet), kcal/day } \\
\hline $\begin{array}{l}\text { Within limits (men } \\
\text { 800-4000; women } \\
500-3500 \text { ) }\end{array}$ & 2913 & 1.00 (ref) & & 1.00 (ref) & - & 1.00 (ref) & - & 1.00 (ref) & - \\
\hline $\begin{array}{l}\text { Beyond limits } \\
\quad(\text { men }<800 \\
\text { or }>4000 \\
\text { women }<500 \\
\text { or }>3500)\end{array}$ & 72 & $0.96(0.60-1.54)$ & 0.877 & $0.67(0.39-1.17)$ & 0.157 & $1.21(0.75-1.94)$ & 0.431 & $1.01(0.58-1.75)$ & 0.985 \\
\hline \multicolumn{10}{|c|}{ Fruit + vegetable consumption, g/day } \\
\hline $\begin{array}{l}\text { Q1 }(\text { men, }<473 \\
\text { women }<544)\end{array}$ & 747 & 1.00 (ref) & - & 1.00 (ref) & - & 1.00 (ref) & - & 1.00 (ref) & - \\
\hline $\begin{array}{r}\text { Q2 (men 473-624; } \\
\text { women 544-698) }\end{array}$ & 746 & $1.13(0.93-1.39)$ & & $1.31(1.03-1.68)$ & & $0.92(0.75-1.13)$ & & $0.96(0.75-1.23)$ & \\
\hline $\begin{array}{r}\text { Q3 (men 625-795; } \\
\text { women 699-886) }\end{array}$ & 746 & $0.84(0.69-1.03)$ & & $0.96(0.73-1.25)$ & & $0.86(0.70-1.06)$ & & $0.91(0.70-1.20)$ & \\
\hline $\begin{array}{l}\text { Q4 (men > 795; } \\
\text { women > 886) }\end{array}$ & 746 & $0.80(0.66-0.99)$ & 0.004 & $0.97(0.72-1.30)$ & 0.417 & $0.73(0.60-0.90)$ & 0.002 & $0.82(0.61-1.10)$ & 0.171 \\
\hline \multicolumn{10}{|c|}{ Meat consumption, g/day } \\
\hline $\begin{array}{r}\text { Q1 }(\text { men }<114 ; \\
\text { women }<105)\end{array}$ & 747 & 1.00 (ref) & - & 1.00 (ref) & - & 1.00 (ref) & - & 1.00 (ref) & - \\
\hline $\begin{array}{l}\text { Q2 (men 114-147; } \\
\text { women 105-137) }\end{array}$ & 746 & $1.30(1.06-1.59)$ & & $1.30(1.03-1.63)$ & & $1.15(0.94-1.41)$ & & $1.10(0.87-1.38)$ & \\
\hline $\begin{array}{l}\text { Q3 (men 148-188; } \\
\text { women 138-174) }\end{array}$ & 747 & $1.19(0.97-1.46)$ & & $1.07(0.85-1.35)$ & & $1.22(1.00-1.49)$ & & $1.07(0.85-1.36)$ & \\
\hline $\begin{array}{l}\text { Q4 }(\text { men > 188; } \\
\text { women > 174) }\end{array}$ & 745 & $1.34(1.09-1.64)$ & 0.015 & $1.14(0.89-1.46)$ & 0.610 & $1.22(1.00-1.50)$ & 0.053 & $0.97(0.76-1.25)$ & 0.745 \\
\hline \multicolumn{10}{|c|}{ Baseline dietary fat intake, $\% \mathrm{E}$, mean (SD) } \\
\hline $\mathrm{Q} 1(<35)$ & 747 & 1.00 (ref) & - & 1.00 (ref) & - & 1.00 (ref) & - & 1.00 (ref) & - \\
\hline Q2 (35-39) & 746 & $1.13(0.93-1.39)$ & & $1.21(0.96-1.53)$ & & $1.05(0.86-1.29)$ & & $1.10(0.87-1.38)$ & \\
\hline Q3 (40-43) & 746 & $1.08(0.88-1.32)$ & & $1.14(0.90-1.44)$ & & $0.95(0.77-1.16)$ & & $0.98(0.78-1.24)$ & \\
\hline $\mathrm{Q} 4(>43)$ & 746 & $1.08(0.88-1.32)$ & 0.573 & $1.28(1.00-1.64)$ & 0.082 & $0.93(0.76-1.13)$ & 0.311 & $1.09(0.85-1.40)$ & 0.665 \\
\hline \multicolumn{10}{|l|}{ Fiber intake, g/day } \\
\hline $\begin{array}{r}\text { Q1 }(\text { men }<20 ; \\
\text { women }<21)\end{array}$ & 747 & 1.00 (ref) & - & 1.00 (ref) & - & 1.00 (ref) & - & 1.00 (ref) & - \\
\hline
\end{tabular}


Table 2 (continued)

\begin{tabular}{|c|c|c|c|c|c|c|c|c|c|}
\hline \multirow[t]{3}{*}{ Baseline characteristics } & \multirow[t]{3}{*}{$n$} & \multicolumn{8}{|c|}{$\begin{array}{l}\text { OR ( } 95 \% \mathrm{CI}) \text { for adherence (increasing } \geq 5 \text { points if baseline }<13 p \text { or any increase if baseline } \geq 13 p)^{1} \text { to the } \\
\text { MedDiet intervention (adherent vs. non-adherent) }{ }^{2}\end{array}$} \\
\hline & & \multicolumn{4}{|l|}{6 month-follow-up } & \multicolumn{4}{|c|}{12 month-follow-up } \\
\hline & & Crude $^{3}$ & $p$ value & Multivariable $^{4}$ & $p$ value & Crude $^{3}$ & $p$ value & Multivariable $^{4}$ & $p$ value \\
\hline $\begin{array}{l}\text { Q2 (men 20-24; } \\
\text { women 21-25) }\end{array}$ & 746 & $0.87(0.71-1.06)$ & & $1.09(0.83-1.43)$ & & $0.98(0.80-1.20)$ & & $1.30(0.99-1.71)$ & \\
\hline $\begin{array}{r}\mathrm{Q} 3(\mathrm{men} 25-30 ; \\
\text { women 26-32) }\end{array}$ & 746 & $0.84(0.68-1.02)$ & & $1.36(0.97-1.88)$ & & $0.87(0.71-1.06)$ & & $1.40(1.00-1.94)$ & \\
\hline $\begin{array}{r}\mathrm{Q} 4(\text { men }>30 \\
\text { women }>32)\end{array}$ & 746 & $0.74(0.60-0.90)$ & 0.004 & $1.62(1.07-2.46)$ & 0.016 & $0.75(0.61-0.92)$ & 0.002 & $1.62(1.07-2.45)$ & 0.039 \\
\hline \multicolumn{10}{|c|}{${ }^{8,11}$ Carbohydrate Quality Index } \\
\hline Q1 (low) & 981 & 1.00 (ref) & - & 1.00 (ref) & - & 1.00 (ref) & - & 1.00 (ref) & - \\
\hline Q2 & 803 & $0.82(0.68-0.99)$ & & $0.96(0.77-1.21)$ & & $0.81(0.67-0.97)$ & & $0.96(0.77-1.21)$ & \\
\hline Q3 & 682 & $0.70(0.58-0.85)$ & & $0.96(0.74-1.26)$ & & $0.67(0.55-0.82)$ & & $0.94(0.72-1.23)$ & \\
\hline Q4 (high) & 519 & $0.61(0.49-1.75)$ & $<0.001$ & $1.00(0.72-1.39)$ & 0.911 & $0.55(0.44-0.68)$ & $<0.001$ & $0.96(0.69-1.34)$ & 0.713 \\
\hline
\end{tabular}

Bold font is used for significant results for multivariate analysis

$C I$ confidence intervals, $C V D$, cardiovascular disease, $D B P$ diastolic blood pressure, $E$ energy, MedDiet Mediterranean diet, $O R$ odds ratio, $M E T$ metabolic equivalent, $Q$ quartile, $R A P A$ rapid assessment of physical activity, $S B P$ systolic blood pressure

For continuous variables, missing data were imputed using simple imputation. For categorical variables, missing data were not imputed and grouped as additional categorical group

${ }^{1}$ Adherence to Mediterranean diet was evaluated using a 17-point scale of adherence to an energy-reduced MedDiet questionnaire (1 point for each item). Participants with an increase of $\geq 5$ points from baseline to follow-up were classified in the "adherent group". Participants with $\geq 13$ points at baseline and positive increase ( $\geq 1$ point) from baseline to follow-up were additionally classified in the "adherent group". Detailed information is provided in Additional File 1: Figure s1

${ }^{2} \mathrm{ORs}<1$ was referred as poorer adherence and ORs $>1$ was referred as better adherence

${ }^{3}$ Crude model implied bivariate logistic regression

${ }^{4}$ Multivariable model implied multivariable-adjusted logistic regression, adjusted for all characteristics displayed in Table 2 with the addition of the 17-item energy-reduced MedDiet score (data available on Additional File 1: Figure s1)

${ }^{5}$ Family history of premature CVD was defined as any immediate family member deceased younger than 55 years for men and 65 years for women

${ }^{6}$ Number of chronic conditions was calculated by summing the following chronic conditions (1 point for each condition): hypertension, obesity, type 2 diabetes, hypercholesterolemia, cancer, and depression)

${ }^{7}$ Self-reported measure of nervousness and/or aggressiveness behavior was reported on a scale from 1 (very low self-perception) to 10 (very high self-perception)

${ }^{8} P$ values for trend were calculated by assigning the median value to each category and treating the resulting variable as continuous

${ }^{9}$ Recruitment year was referred to the period (years) in which participants were recruited, from the date of the first recruited participant $(9 / 05 / 2013)$ to the date of the last recruited participant (10/31/2016)

${ }^{10}$ Total workload of center was measured as the number of participants per center

${ }^{11}$ Carbohydrate Quality Index was referred to the quality of dietary carbohydrate intake and was constructed upon four carbohydrate quality domains: total dietary fiber intake ( $\mathrm{g} / \mathrm{day}$ ), glycemic index, ratio of carbohydrates from whole grains to carbohydrates from total grains (whole grains + refined grains or their products), and ratio of carbohydrates from solid foods to total carbohydrates (solid carbohydrates + liquid carbohydrates). Quartiles of carbohydrate Quality Index (score) were: Q1: <9; Q2: 9-10; Q3: 11-12; Q4:>12

to the 17-item erMedDiet score. This circumstance was also observed when conducting the main analysis.

\section{Discussion}

The PREDIMED-Plus is an intensive nutritional intervention based on major long-term dietary behavioral change aimed to improve participants' health outcomes, including their risk of cardiovascular events, which also includes regular physical activity and weight-loss goals [31]. We longitudinally examined baseline characteristics related to the attainment of successful dietary behavior changes. The most consistent factors of successful compliance were high baseline perceived self-efficacy to modify diet and high baseline fiber intake. In contrast, the presence of depression and multiple chronic diseases were factors independently associated with poorer compliance. 
Table 3 Association between each individual chronic condition (hypertension, obesity, type 2 diabetes, hypercholesterolemia, cancer, and depression) and good adherence ${ }^{1}$ (increasing $\geq 5$ points if base- line $<13$ or any increase if baseline $\geq 13$ ) to the MedDiet at 6 and 12 months in the active intervention group of the PREDIMED-Plus trial $(n=2,985)$

\begin{tabular}{|c|c|c|c|c|c|c|c|c|c|}
\hline \multirow{3}{*}{$\begin{array}{l}\text { Baseline } \\
\text { characteris- } \\
\text { tics }\end{array}$} & \multirow[t]{3}{*}{$n$} & \multicolumn{8}{|c|}{$\begin{array}{l}\text { OR }(95 \% \mathrm{CI}) \text { for adherence (increasing } \geq 5 \text { points if baseline }<13 p \text { or any increase if baseline } \geq 13 p)^{1} \text { to the MedDiet } \\
\text { intervention (adherent vs. non-adherent) })^{2}\end{array}$} \\
\hline & & \multicolumn{4}{|l|}{6 month-follow-up } & \multicolumn{4}{|c|}{12 month-follow-up } \\
\hline & & Crude $^{3}$ & $p$ value & Multivariable $^{4}$ & $p$ value & Crude $^{3}$ & $p$ value & Multivariable $^{4}$ & $p$ value \\
\hline \multicolumn{10}{|c|}{ Health-related characteristics } \\
\hline \multicolumn{10}{|c|}{ Hypertension } \\
\hline No & 488 & 1.00 (ref) & - & 1.00 (ref) & - & 1.00 (ref) & - & 1.00 (ref) & - \\
\hline Yes & 2497 & $0.89(0.74-1.09)$ & 0.260 & $0.81(0.65-1.01)$ & 0.063 & $1.03(0.84-1.24)$ & 0.802 & $0.95(0.76-1.19)$ & 0.655 \\
\hline \multicolumn{10}{|l|}{ Obesity } \\
\hline No & 815 & 1.00 (ref) & & 1.00 (ref) & - & 1.00 (ref) & - & 1.00 (ref) & - \\
\hline Yes & 2170 & $1.00(0.85-1.18)$ & 0.965 & $0.81(0.65-1.01)$ & 0.067 & $1.08(0.92-1.27)$ & 0.334 & $1.10(0.87-1.37)$ & 0.425 \\
\hline \multicolumn{10}{|c|}{ Type 2 diabetes } \\
\hline No & 2154 & 1.00 (ref) & & 1.00 (ref) & - & 1.00 (ref) & - & 1.00 (ref) & - \\
\hline Yes & 831 & $0.65(0.55-0.76)$ & $<0.001$ & $0.69(0.55-0.86)$ & 0.001 & $0.72(0.61-0.85)$ & $<0.001$ & $0.82(0.66-1.02)$ & 0.080 \\
\hline \multicolumn{10}{|c|}{ Hypercholesterolemia } \\
\hline No & 897 & 1.00 (ref) & - & 1.00 (ref) & - & 1.00 (ref) & - & 1.00 (ref) & - \\
\hline Yes & 2088 & $0.89(0.76-1.04)$ & 0.154 & $0.91(0.76-1.08)$ & 0.288 & $0.99(0.84-1.15)$ & 0.853 & $1.01(0.85-1.21)$ & 0.876 \\
\hline \multicolumn{10}{|l|}{ Cancer } \\
\hline No & 2765 & 1.00 (ref) & - & 1.00 (ref) & - & 1.00 (ref) & - & 1.00 (ref) & - \\
\hline Yes & 220 & $0.93(0.70-1.22)$ & 0.582 & $0.96(0.70-1.31)$ & 0.791 & $0.98(0.74-1.29)$ & 0.867 & $1.01(0.74-1.39)$ & 0.932 \\
\hline \multicolumn{10}{|l|}{ Depression } \\
\hline No & 2408 & 1.00 (ref) & - & 1.00 (ref) & - & 1.00 (ref) & - & 1.00 (ref) & - \\
\hline Yes & 577 & $0.80(0.67-0.96)$ & 0.017 & $0.80(0.64-0.99)$ & 0.036 & $0.73(0.61-0.87)$ & 0.001 & $0.71(0.57-0.88)$ & 0.002 \\
\hline
\end{tabular}

Bold font is used for significant results for multivariate analysis

CI confidence intervals, MedDiet Mediterranean diet, OR odds ratios

${ }^{1}$ Adherence to Mediterranean diet was evaluated using a 17-point scale of adherence to an energy-reduced MedDiet questionnaire (1 point for each item). Participants with an increase of $\geq 5$ points from baseline to follow-up were classified in the "adherent group". Participants with $\geq 13$ points at baseline and positive increase ( $\geq 1$ point) from baseline to follow-up were additionally classified in the "adherent group". Detailed information is provided in Additional File 1: Figure s1

${ }^{2} \mathrm{ORs}<1$ was referred as poorer adherence and ORs $>1$ was referred as better adherence

${ }^{3}$ Multivariable model implied multivariable-adjusted logistic regression, adjusted for the same predictors than the logistic model of Table 2 with the addition of hypertension, obesity, type 2 diabetes, hypercholesterolemia, cancer, and depression, and the exclusion of chronic conditions

\section{Socio-demographics}

Previous studies have reported inconclusive results regarding the association between adherence to the MedDiet and socio-demographic characteristics, including sex [9-11, 13, 15, 32-34], or working status [9, 10, 34, 35]. However, married individuals compared to single people seem to respond better to intended dietary changes in previous studies [11, 34], probably because the greater predominance of structured and routine dietary habits among married persons. In our study, we did not find any consistent pattern.

\section{Health-related characteristics}

Individuals with multiple chronic conditions may benefit the most from adhering to a healthy dietary pattern. For instance, the MedDiet has demonstrated numerous positive effects on preventing chronic diseases and improving health outcomes, including type 2 diabetes and depression $[8,36]$. Nevertheless, in our study, we found that participants with diabetes and those with a higher number of chronic conditions were less likely to attain high adherence. Prior findings in other studies showed similar results. For example, participants with obesity have been reported to show poorer MedDiet adherence [12], whereas having diabetes and suffering from a greater number of chronic conditions were independent factors of lower compliance $[9,10]$. Additionally, our results suggested that a diagnosis of depression at baseline is a strong barrier to modify dietary behavior. Similar findings of poor behavior change among participants with depression have been observed in long-term dietary interventions [37], dietary weight-loss 


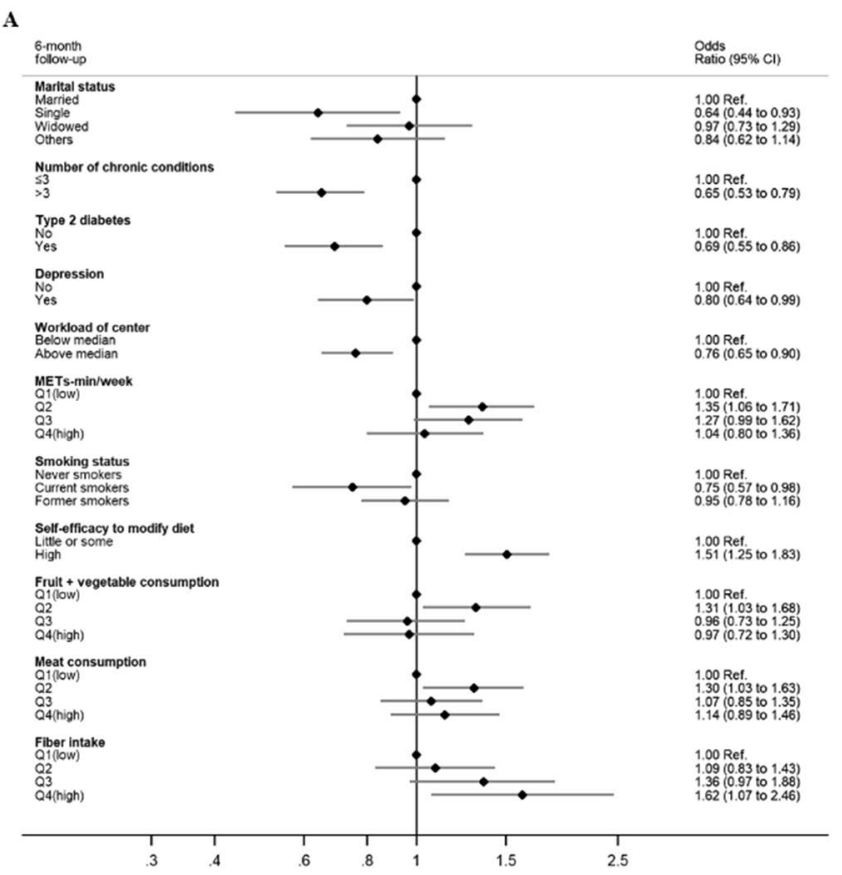

Fig. 2 Independent factors of good adherence. Odds ratios (OR) and 95\% confidence intervals (95\% CI) of good adherence (increasing $\geq 5$ points if baseline $<13$ or any increase if baseline $\geq 13$ ) to the MedDiet intervention at A 6 months and B 12 months of follow-up in the active intervention group of the PREDIMED-Plus trial $(n=2,985)$. Adherence to Mediterranean diet was evaluated using a 17-point scale of adherence to an energy-reduced MedDiet questionnaire (1 point for each item). Participants with an increase of $\geq 5$ points from baseline to follow-up were classified in the "adherent group". Participants with $\geq 13$ points at baseline and positive increase ( $\geq 1$ point) from baseline to follow-up were additionally classified in the "adherent

trials [38], and prevention programs for individuals with metabolic syndrome [39]. Moreover, depression has been associated with poorer attendance and early drop-outs in behavioral trials [40]. Potential explanations for these findings may rely on the inherent psychological characteristics of individuals with depression and on the established unhealthy dietary habits that may lead individuals with depression and other chronic conditions to their current health status; nutrition myths or misconceptions related to their diseases [41], and excessive nutritional information received from health care professionals and other sources such as family, friends, or websites [42], may additionally explain these findings. Based on these associations, future dietary behavioral RCTs should carefully collect information about participants' psychological attributes at baseline, as this information is frequently sub-optimally collected [43]. Exclusion of individuals with depression would allow to identify probable candidates for early dropouts and low compliance, and ensure a significant contrast between the intervention and control arms of future trials.
B

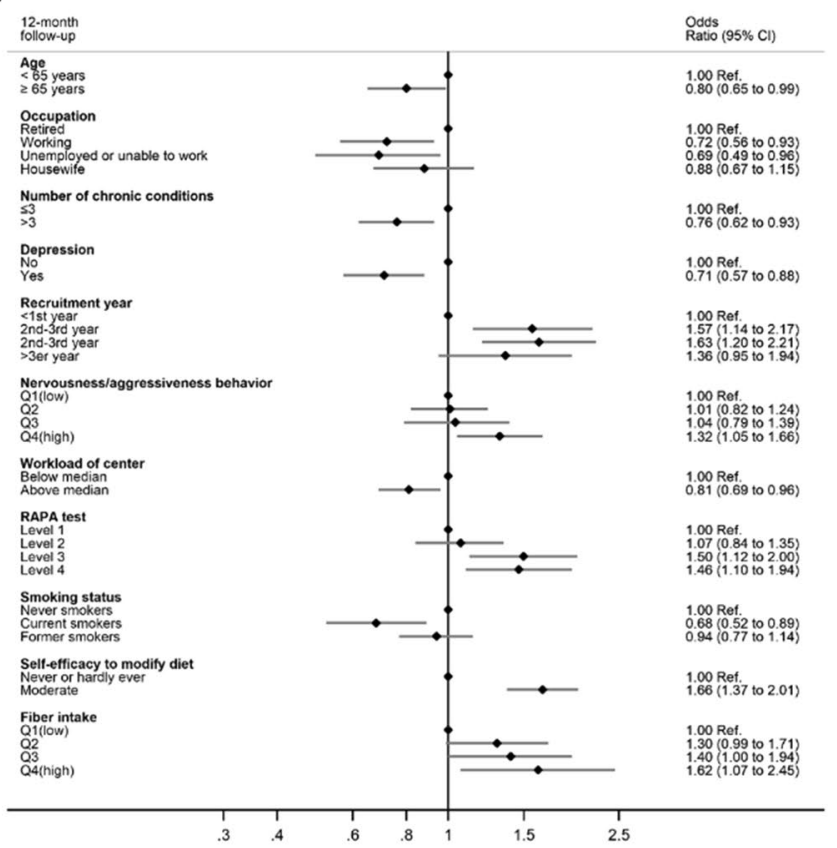

group". Detailed information is provided in Additional File 1: Figure s1. Dietary fat intake was expressed as \% of energy. Fiber intake was expressed in $\mathrm{g} /$ day. Recruitment year was referred to the period (years) in which participants were recruited, from the date of the first recruited participant $(9 / 05 / 2013)$ to the date of the last recruited participant (10/31/2016). Nervousness/aggressiveness behavior was selfreported on a scale from 1 (very low self-perception) to 10 (very high self-perception). RAPA test levels were categorized as: level 1 (sedentary or under-active), level 2 (under-active regular - light activities), level 3 (under-active regular), and level 4 (active). Workload of center was measured as total number of persons-years of follow-up
On the other hand, careful design of RCTs specifically targeting these participants with depression may be a desirable approach so that they could also beneficiate from tailored dietary interventions, but they will need very specific and particularly intensive intervention protocols. A more intense and specific dietary counseling with adapted information and personalized messages for individuals with chronic conditions is also highly warranted.

\section{Lifestyle and behavior}

In our study, high self-efficacy, a social cognitive theory component, was an important predictor of better compliance to the erMedDiet. According to Bandura et al. [44], an individual's beliefs according to his/her abilities determine certain behaviors. In this case, participants' beliefs regarding personal success on the desired outcome (dietary changes) may determine their effort level. Hence, participants with high perceived self-efficacy are expected to pursue dietary modifications until successful dietary change is achieved. 
Consistent with our findings, high self-efficacy has been a promising predictor in weight-loss interventions and physical activity among overweight/obese populations [45]. Moreover, long-term maintenance of high self-efficacy has been associated with greater weight loss [46]. This evidence emphasizes the importance of collecting this information at baseline and incorporating specific strategies to maintain a high self-efficacy level throughout the follow-up period of the interventions. Negotiated goal setting, continuous persuasion, permanent performance feedback, shared decisionmaking, alternatives to overcome struggles faced by participants to improve their diets, and problem-solving strategies are different approaches which may improve and maintain self-efficacy along the trial. These strategies have been key in previous RCTs, such as the PREDIMED, to successfully improve participants' adherence to the MedDiet in the intervention groups [8].

\section{Dietary characteristics}

Participants with a poorer baseline adherence to the erMedDiet score, had greater room for improvement whereas participants with higher adherence at baseline may face a ceiling effect. Therefore, it is not surprising to find better achievements among those with poorer scores at baseline. Interestingly, we found that higher fiber intake was a factor independently associated with better dietary changes. In previous studies, fiber intake resulted a robust predictor of weight loss and beneficial food-related behavioral changes $[47,48]$. Participants with high fiber intake at baseline may be more health conscious and they may be more likely to better adapt to fiber-rich food patterns such, as the Mediterranean diet than individuals with poorer baseline fiber intake. This finding was observed after adjustment for the baseline adherence to the 17-item erMedDiet score. This is important, given that participants with poorer adherence to the MedDiet usually tend to have lower fiber intake, as it was the case in our study.

The current study has some limitations. First, information about the participants' diet and health conditions was selfreported, and recall bias and misreporting may be present when using self-reported information. Nevertheless, their self-reported changes were paralleled by objective changes in cardiovascular risk factors as reported elsewhere [17, 30]. Second, the observational nature of the study limits causal inferences. Third, although we tested several characteristics to predict behavior change and adjusted for a wide array of potential confounding factors, failure to control for other factors may be possible and we cannot exclude residual confounding. Nevertheless, we examined multiple potential factors chosen according to the existing literature and some of our previous studies. And fourth, the PREDIMEDPlus includes an overweight/obese community-dwelling population with metabolic syndrome which is not representative of the general population. However, this population is becoming more predominant in developed and developing countries, increasing the actual practical interest of our findings. Despite the aforementioned limitations, the strength of our study relies on the evaluation of a high number of baseline candidate factors, the inclusion of several sensitivity analyses that corroborated our findings, and the use of high-quality prospective data with a very high retention rate from one of the largest nutritional trials, the PREDIMEDPlus trial.

\section{Conclusions}

The present study provides a better understanding of factors associated with successful compliance to a dietary intervention. Recruitment of individuals highly motivated to change their diet and of those who follow a fiber-rich dietary pattern but even so, they poorly adhere at baseline to the intended diet would potentially increase the needed contrast between the arms of a dietary intervention trial. Participants with multiple chronic conditions, particularly depression, should receive tailored protocols and specific attention, because they are not likely to respond to conventional interventions. Future studies should investigate strategies to promote better compliance among those individuals with features which predict poor compliance to the intended dietary behavior changes.

Supplementary Information The online version contains supplementary material available at https://doi.org/10.1007/s00394-021-02697-8.

Acknowledgements More extensive acknowledgments are included in the online Additional File 1.

Author contributions CIF-L, ET, PB-C, JS-S, DC, MF, JAM, AMA-G, JW, JV, DR, JLM, RE, FJT, JL, JLS-M, AB-C, JAT, VMS, XP, MD-R, PM-M, JV, ER, CV, LD, BS, JG, JVS, OC, MAZ, LT-S, NP-F, AO-C, MM, AG-R, ES, RMB-L, JMS-L, ZV-R, JM, CO-A, AG, CR, LG-G, MR-C, NB-T, HS, and MAM-G were involved in the study design and conducted the research; CIF-L and MAM-G analyzed the data; CIF-L and MAM-G wrote the manuscript; CIF-L, ET, PB-C, JS-S, DC, MF, JAM, AMA-G, JW, JV, DR, JLM, RE, FJT, JL, JLS-M, AB-C, JAT, VMS, XP, MD-R, PM-M, JV, ER, CV, LD, BS, JG, JVS, OC, MAZ, LT-S, NP-F, AO-C, MM, AG-R, ES, RMB-L, JMS-L, ZV-R, JM, CO-A, AG, CR, LG-G, MR-C, NB-T, HS, and MAM-G revised the manuscript and provided critical edits. MAM-G and CIF-L had primary responsibility for final content. All authors read and approved the final manuscript.

Funding Open Access funding provided thanks to the CRUE-CSIC agreement with Springer Nature. The PREDIMED-Plus trial was supported by the European Research Council (advanced research grant 2014-2019, 340918 to MAM-G as PI) and by the official Spanish Institutions for funding scientific biomedical research, CIBER Fisiopatología de la Obesidad y Nutrición (CIBERobn) and Instituto 
de Salud Carlos III (ISCIII), through the Fondo de Investigación en Salud (FIS), which is cofunded by the European Regional Development Fund coordinated by J.S.-S. and J.V., including the following projects: PI13/00673, PI13/00492, PI13/00272, PI13/01123, PI13/00462, PI13/00233, PI13/02184, PI13/00728, PI13/01090, PI13/01056, PI14/01722, PI14/00636, PI14/00618, PI14/00696, PI14/01206, PI14/01919, PI14/00853, PI14/01374, PI14/00972, PI14/00728, PI14/01471, PI16/00473, PI16/00662, PI16/01873, PI16/01094, PI16/00501, PI16/00533, PI16/00381, PI16/00366, PI16/01522, PI16/01120, PI17/00764, PI17/01183, PI17/00855, PI17/01347, PI17/00525, PI17/01827, PI17/00532, PI17/00215, PI17/01441, PI17/00508, PI17/01732, PI17/00926, PI19/00957, PI19/00386, PI19/00309, PI19/01032, PI19/00576, PI19/00017, PI19/01226, PI19/00781, PI19/01560, PI19/01332, PI20/01802, PI20/00138, PI20/01532, PI20/00456, PI20/00339, PI20/00557, PI20/00886, and PI20/01158); the Especial Action Project entitled Implementación y evaluación de una intervención intensiva sobre la actividad física Cohorte PREDIMED-Plus grant, the Recercaixa grant (2013ACUP00194), Grants from the Consejería de Salud de la Junta de Andalucía, a grant from the Generalitat Valenciana, a SEMERGEN grant, and funds from the European Regional Development Fund (CB06/03; CB12/03), The International Nut \& Dried Fruit; and the AstraZeneca Young Investigators Award in Category of Obesity and T2D 2017 to DR. J.S.-S. is partially supported by ICREA under the ICREA Academia programme. None of the funding sources took part in the design, collection, analysis, or interpretation of the data or in the decision to submit the manuscript for publication.

Availability of data and material There are restrictions on the availability of data for the PREDIMED-Plus trial, due to the signed consent agreements around data sharing, which only allow access to external researchers for studies following the project purposes. Requestors wishing to access the PREDIMED-Plus trial data used in this study can make a request to the PREDIMED-Plus trial Steering Committee chair: predimed_plus_scommitte@googlegroups.com. The request will then be passed to members of the PREDIMED-Plus Steering Committee for deliberation.

\section{Declarations}

Conflict of interest The authors declare that there is no conflict of interest regarding the publication of this article.

Ethics approval The research ethics committees of all centers approved the study protocol.

Consent to participate All participants provided written informed consent.

\section{Consent for publication No applicable.}

Open Access This article is licensed under a Creative Commons Attribution 4.0 International License, which permits use, sharing, adaptation, distribution and reproduction in any medium or format, as long as you give appropriate credit to the original author(s) and the source, provide a link to the Creative Commons licence, and indicate if changes were made. The images or other third party material in this article are included in the article's Creative Commons licence, unless indicated otherwise in a credit line to the material. If material is not included in the article's Creative Commons licence and your intended use is not permitted by statutory regulation or exceeds the permitted use, you will need to obtain permission directly from the copyright holder. To view a copy of this licence, visit http://creativecommons.org/licenses/by/4.0/.

\section{References}

1. Pan A, Lin X, Hemler E, Hu FB (2018) Diet and cardiovascular disease: advances and challenges in population-based studies. Cell Metab 27:489-496. https://doi.org/10.1016/j.cmet.2018.02.017

2. Satija A, Stampfer MJ, Rimm EB et al (2018) Perspective: are large, simple trials the solution for nutrition research? Adv Nutr 9:378-387. https://doi.org/10.1093/advances/nmy030

3. Howard BV, Van Horn L, Hsia J et al (2006) Low-fat dietary pattern and risk of cardiovascular disease: the Women's Health Initiative randomized controlled dietary modification trial. JAMA 295:655-666. https://doi.org/10.1001/jama.295.6.655

4. Prentice RL, Caan B, Chlebowski RT et al (2006) Low-fat dietary pattern and risk of invasive breast cancer: the Women's Health Initiative randomized controlled dietary modification trial. JAMA 295:629-642. https://doi.org/10.1001/jama.295.6.629

5. Orloff JN, Aronne LJ, Shukla AP (2018) The challenge of meeting prescribed carbohydrate intake goals in low-carbohydrate diet studies. Am J Clin Nutr 107:673-674. https://doi.org/10.1093/ ajen/nqy023

6. Kjelsberg MO (1982) Multiple risk factor intervention trial: risk factor changes and mortality results. JAMA 248:1465-1477. https://doi.org/10.1001/jama.1982.03330120023025

7. Willett WC, Stampfer MJ (1990) Dietary fat and cancer: another view. Cancer Causes Control 1:103-109. https://doi.org/10.1007/ BF00053190

8. Fernández-Lázaro CI, Ruiz-Canela M, Martínez-González MÁ (2021) Deep dive to the secrets of the PREDIMED trial. Curr Opin Lipidol 32:62-69. https://doi.org/10.1097/MOL.00000 00000000731

9. Zazpe I, Estruch R, Toledo E et al (2010) Predictors of adherence to a Mediterranean-type diet in the PREDIMED trial. Eur J Nutr 49:91-99. https://doi.org/10.1007/s00394-009-0053-7

10. Downer MK, Gea A, Stampfer M et al (2016) Predictors of short- and long-term adherence with a Mediterranean-type diet intervention: the PREDIMED randomized trial. Int J Behav Nutr Phys Act 13:67. https://doi.org/10.1186/s12966-016-0394-6

11. Hu EA, Toledo E, Diez-Espino J et al (2013) Lifestyles and risk factors associated with adherence to the mediterranean diet: a baseline assessment of the PREDIMED trial. PLoS ONE 8:e60166. https://doi.org/10.1371/journal.pone.0060166

12. Patino-Alonso MC, Recio-Rodríguez JI, Belio JFM et al (2014) Factors associated with adherence to the Mediterranean diet in the adult population. J Acad Nutr Diet 114:583-589. https://doi. org/10.1016/j.jand.2013.07.038

13. Ruggiero E, Di Castelnuovo A, Costanzo S et al (2019) Socioeconomic and psychosocial determinants of adherence to the Mediterranean diet in a general adult Italian population. Eur J Public Health 29:328-335. https://doi.org/10.1093/eurpub/ cky 127

14. Peng W, Goldsmith R, Berry EM (2016) Demographic and lifestyle factors associated with adherence to the Mediterranean diet in relation to overweight/obesity among Israeli adolescents: findings from the Mabat Israeli national youth health and nutrition survey. Public Health Nutr 20:883-892. https://doi.org/10.1017/ S1368980016002779

15. Grosso G, Marventano S, Buscemi S et al (2013) Factors associated with adherence to the Mediterranean diet among adolescents living in Sicily, southern Italy. Nutrients 5:4908-4923. https://doi. org/10.3390/nu5124908

16. Martínez-González MA, Buil-Cosiales P, Corella D et al (2019) Cohort Profile: Design and methods of the PREDIMED-Plus randomized trial. Int J Epidemiol 48:387-388o. https://doi.org/10. 1093/ije/dyy225 
17. Sayón-Orea C, Razquin C, Bulló M et al (2019) Effect of a Nutritional and Behavioral Intervention on energy-reduced mediterranean diet adherence among patients with metabolic syndrome: interim analysis of the PREDIMED-plus randomized clinical trial. JAMA 322:1486-1499. https://doi.org/10.1001/jama.2019.14630

18. Schröder H, Cárdenas-Fuentes G, Martínez-González MA et al (2018) Effectiveness of the physical activity intervention program in the PREDIMED-Plus study: a randomized controlled trial. Int J Behav Nutr Phys Act 15:110. https://doi.org/10.1186/ s12966-018-0741-x

19. World Health Organization (2021) Physical activity and older adults. https://www.who.int/ncds/prevention/physical-activity/ factsheet_olderadults/en. Accessed 1 Mar 2020

20. Alberti KGMM, Eckel RH, Grundy SM et al (2009) Harmonizing the metabolic syndrome: a joint interim statement of the international diabetes federation task force on epidemiology and prevention; National heart, lung, and blood institute; American heart association; World heart federation; International. Circulation 120:1640-1645. https://doi.org/10.1161/CIRCULATIONAHA. 109.192644

21. Schröder H, Zomeño MD, Martínez-González MA et al (2021) Validity of the energy-restricted Mediterranean Diet Adherence Screener. Clin Nutr 40:4971-4979. https://doi.org/10.1016/j.clnu. 2021.06.030

22. de la Fuente-Arrillaga C, Vázquez Ruiz Z, Bes-Rastrollo M et al (2010) Reproducibility of an FFQ validated in Spain. Public Health Nutr 13:1364-1372. https://doi.org/10.1017/S136898000 9993065

23. Fernández-Ballart JD, Piñol JL, Zazpe I et al (2010) Relative validity of a semi-quantitative food-frequency questionnaire in an elderly Mediterranean population of Spain. Br J Nutr 103:18081816. https://doi.org/10.1017/S0007114509993837

24. Moreiras O, Carbajal A, Cabrera L, Cuadrado C (2011) Tablas de Composición de Alimentos (Food Composition Tables), 15th edn. Pirámide, Madrid

25. Mataix-Verdú J, García-Diaz L, Manas M et al (2003) Tabla de Composición de Alimentos (Spanish Food Composition Tables), 4th edn. Universidad de Granada Press, Granada

26. Topolski TD, LoGerfo J, Patrick DL et al (2006) The rapid assessment of physical activity (RAPA) among older adults. Prev Chronic Dis 3:A118

27. Molina L, Sarmiento M, Peñafiel J et al (2017) Validation of the regicor short physical activity questionnaire for the adult population. PLoS ONE 12:e0168148. https://doi.org/10.1371/journal. pone. 0168148

28. Martínez-González MA, López-Fontana C, Varo JJ et al (2005) Validation of the Spanish version of the physical activity questionnaire used in the Nurses' Health Study and the Health Professionals' Follow-up Study. Public Health Nutr 8:920-927. https://doi. org/10.1079/PHN2005745

29. Willet WC (2013) Nutritional epidemiology, 3rd edn. Oxford University Press, New York

30. Martínez-González MA, Fernandez-Lazaro CI, Toledo E et al (2019) Carbohydrate quality changes and concurrent changes in cardiovascular risk factors: a longitudinal analysis in the PREDIMED-plus randomized trial. Am J Clin Nutr 111:291-306. https://doi.org/10.1093/ajen/nqz298

31. Martínez-González MÁ, Corella D, Salas-Salvadó J et al (2012) Cohort profile: design and methods of the PREDIMED study. Int J Epidemiol 41:377-385. https://doi.org/10.1093/ije/dyq250

32. Sánchez-Villegas A, Martínez JA, De Irala J, Martínez-González MA (2002) Determinants of the adherence to an "a priori" defined Mediterranean dietary pattern. Eur J Nutr 41:249-257. https://doi. org/10.1007/s00394-002-0382-2

33. González CA, Argilaga S, Agudo A et al (2002) Diferencias sociodemográficas en la adhesión al patrón de dieta mediterránea en poblaciones de España (sociodemographic differences in adherence to the Mediterranean dietary pattern in Spanish populations). Gac Sanit 16:214-221. https://doi.org/10.1016/S0213-9111(02) 71664-6

34. Jurado D, Burgos-Garrido E, Diaz FJ et al (2012) Adherence to the Mediterranean dietary pattern and personality in patients attending a primary health center. J Acad Nutr Diet 112:887-891. https://doi.org/10.1016/j.jand.2012.02.026

35. Marventano S, Godos J, Platania A et al (2018) Mediterranean diet adherence in the Mediterranean healthy eating, aging and lifestyle (MEAL) study cohort. Int J Food Sci Nutr 69:100-107. https:// doi.org/10.1080/09637486.2017.1332170

36. Sánchez-Villegas A, Delgado-Rodríguez M, Alonso A et al (2009) Association of the Mediterranean dietary pattern with the incidence of depression: the Seguimiento Universidad de Navarra/ University of Navarra follow-up (SUN) Cohort. Arch Gen Psychiatry 66:1090-1098. https://doi.org/10.1001/archgenpsychiatry. 2009.129

37. Wang JB, Pierce JP, Ayala GX et al (2015) Baseline depressive symptoms, completion of study assessments, and behavior change in a long-term dietary intervention among breast cancer survivors. Ann Behav Med 49:819-827. https://doi.org/10.1007/ s12160-015-9716-1

38. Somerset SM, Graham L, Markwell K (2011) Depression scores predict adherence in a dietary weight loss intervention trial. Clin Nutr 30:593-598. https://doi.org/10.1016/j.clnu.2011.04.004

39. Susin N, De Melo BR, Ludwig MWB et al (2016) Predictors of adherence in a prevention program for patients with metabolic syndrome. J Health Psychol 21:2156-2167. https://doi.org/10. $1177 / 1359105315572451$

40. Moroshko I, Brennan L, O’Brien P (2011) Predictors of dropout in weight loss interventions: a systematic review of the literature. Obes Rev 12:912-934. https://doi.org/10.1111/j.1467-789X.2011. 00915. $\mathrm{x}$

41. Lesser LI, Mazza MC, Lucan SC (2015) Nutrition myths and healthy dietary advice in clinical practice. Am Fam Physician 91:634-638

42. Ball L, Davmor R, Leveritt $M$ et al (2016) Understanding the nutrition care needs of patients newly diagnosed with type 2 diabetes: a need for open communication and patient-focussed consultations. Aust J Prim Health 22:416-422. https://doi.org/10. 1071/PY15063

43. Somerset SM, Markwell K, Al-Foraih M (2013) A systematic review of baseline psychosocial characterisation in dietary randomised controlled trials for weight loss. Eur J Clin Nutr 67:697702. https://doi.org/10.1038/ejen.2013.77

44. Bandura A (1997) Self-efficacy: the exercise of control. W.H. Freeman and Company, New York

45. Teixeira PJ, Carraça EV, Marques MM et al (2015) Successful behavior change in obesity interventions in adults: a systematic review of self-regulation mediators. BMC Med 13:1-16. https:// doi.org/10.1007/s10865-007-9135-2

46. Warziski MT, Sereika SM, Styn MA et al (2008) Changes in selfefficacy and dietary adherence: the impact on weight loss in the PREFER study. J Behav Med 31:81-92. https://doi.org/10.1007/ s10865-007-9135-2

47. Miketinas DC, Bray GA, Beyl RA et al (2019) Fiber intake predicts weight loss and dietary adherence in adults consuming calorie-restricted diets: the POUNDS lost (Preventing Overweight Using Novel Dietary Strategies) study. J Nutr 149:1742-1748. https://doi.org/10.1093/JN/NXZ117

48. Hiel S, Bindels LB, Pachikian BD et al (2019) Effects of a diet based on inulin-rich vegetables on gut health and nutritional behavior in healthy humans. Am J Clin Nutr 109:1683-1695. https://doi.org/10.1093/AJCN/NQZ001 


\section{Authors and Affiliations}

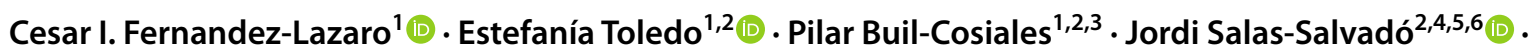
Dolores Corella ${ }^{2,7} \cdot$ Montserrat Fitó ${ }^{2,8}$. J. Alfredo Martínez ${ }^{2,9,10}$. Ángel M. Alonso-Gómez ${ }^{2,11}$ • Julia Wärnberg ${ }^{2,12}$. Jesús Vioque ${ }^{13,14}$ - Dora Romaguera2,15 . José López-Miranda 2,16 Ramon Estruch $^{2,17}$ • Francisco J. Tinahones ${ }^{2,18}$. José Lapetra ${ }^{2,19}$. Luís Serra-Majem ${ }^{2,20}$. Aurora Bueno-Cavanillass ${ }^{13,21}$. Josep A. Tur ${ }^{2,22}$. Vicente Martín Sánchez ${ }^{13,23}$.

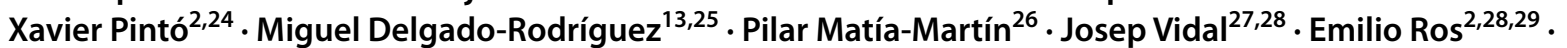
Clotilde Vázquez $^{30}$ - Lidia Daimiel ${ }^{31}$ - Beatriz SanJulián ${ }^{1}$ · Jesús F. García-Gavilán ${ }^{2,4}$ • Jose V. Sorlí ${ }^{2,7}$. Olga Castañer ${ }^{2,8}$. M. Ángeles Zulet ${ }^{2,10}$ • Lucas Tojal-Sierra ${ }^{2,11}$. Napoleón Pérez-Farinós ${ }^{2,12}$ - Alejandro Oncina-Canovas ${ }^{14}$. Manuel Moñino ${ }^{2,15} \cdot$ Antonio Garcia-Rios ${ }^{2,16} \cdot$ Emilio Sacanella $^{2,17} \cdot$ Rosa M. Bernal-Lopez ${ }^{2,18}$. José Manuel Santos-Lozano ${ }^{2,19} \cdot$ Zenaida Vázquez-Ruiz ${ }^{1,2}$. Jananee Muralidharan ${ }^{2,4} \cdot$ Carolina Ortega-Azorín ${ }^{2,7}$. Alberto Goday ${ }^{2,8}$. Cristina Razquin ${ }^{1,2}$ - Leire Goicolea-Güemez ${ }^{2,11} \cdot$ Miguel Ruiz-Canela $^{1,2}$ - Nerea Becerra-Tomás ${ }^{2,4,6}$. Helmut Schröder ${ }^{2,8} \cdot$ Miguel A. Martínez González ${ }^{1,2,32}$ [D · for the PREDIMED-Plus investigators

1 Department of Preventive Medicine and Public Health, NavarraUniversity of Navarra, IdiSNA, C/ Irunlarrea, 31008 Pamplona, Spain

2 Centro de Investigación Biomédica en Red Fisiopatología de la Obesidad y la Nutrición (CIBEROBN), Institute of Health Carlos III, Madrid, Spain

3 Servicios de Atención Primaria, Navarra Regional Health Service (Osasunbidea), IdiSNA, Pamplona, Spain

4 Universitat Rovira I Virgili, Departament de Bioquímica i Biotecnologia, Unitat de Nutrició Humana, Reus, Spain

5 Nutrition Unit, University Hospital of Sant Joan de Reus, Reus, Spain

6 Institut d'Investigació Sanitària Pere Virgili (IISPV), Reus, Spain

7 Department of Preventive Medicine, University of Valencia, Valencia, Spain

8 Unit of Cardiovascular Risk and Nutrition, Institut Hospital del Mar de Investigaciones Médicas Municipal d’Investigació Médica (IMIM), Barcelona, Spain

9 Department of Nutrition, Food Sciences, and Physiology, Center for Nutrition Research, University of Navarra, Pamplona, Spain

10 Cardiometabolic Nutrition Group, IMDEA Food, CEI UAM + CSIC, Madrid, Spain

11 Bioaraba Health Research Institute, Cardiovascular, Respiratory and Metabolic Area, Osakidetza Basque Health Service, Araba University Hospital, University of the Basque Country UPV/EHU, Vitoria-Gasteiz, Spain

12 Epi-Phaan Research Group, School of Health Sciences, Universidad de Málaga, Instituto de Investigación Biomédica de Málaga (IBIMA), 29071 Málaga, Spain

13 CIBER de Epidemiología y Salud Pública (CIBERESP), Instituto de Salud Carlos III, Madrid, Spain

14 Instituto de Investigación Sanitaria y Biomédica de Alicante (ISABIAL-UMH), Alicante, Spain

15 Health Research Institute of the Balearic Islands (IdISBa), Palma de Mallorca, Spain
16 Department of Internal Medicine, Maimonides Biomedical Research Institute of Cordoba (IMIBIC), Reina Sofia University Hospital, University of Cordoba, Cordoba, Spain

17 Department of Internal Medicine, Institut d'Investigacions Biomèdiques August Pi Sunyer (IDIBAPS), Hospital Clinic, University of Barcelona, Barcelona, Spain

18 Department of Endocrinology, Virgen de la Victoria Hospital, Instituto de Investigación Biomédica de Málaga (IBIMA), University of Málaga, Málaga, Spain

19 Department of Family Medicine, Research Unit, Distrito Sanitario Atención Primaria Sevilla, Sevilla, Spain

20 Research Institute of Biomedical and Health Sciences (IUIBS), University of Las Palmas de Gran Canaria and Centro Hospitalario Universitario Insular Materno Infantil (CHUIMI), Canarian Health Service, Las Palmas de Gran Canaria, Spain

21 Department of Preventive Medicine and Public Health, University of Granada, Granada, Spain

22 Research Group on Community Nutrition and Oxidative Stress, University of Balearic Islands-IUNICS and IDISBA, Palma de Mallorca, Spain

23 Institute of Biomedicine (IBIOMED), University of León, León, Spain

24 Lipids and Vascular Risk Unit, Internal Medicine, Hospital Universitario de Bellvitge, Hospitalet de Llobregat,, Barcelona, Spain

25 Division of Preventive Medicine, Faculty of Medicine, University of Jaén, Jaén, Spain

26 Department of Endocrinology and Nutrition, Instituto de Investigación Sanitaria Hospital Clínico San Carlos (IdISSC), Madrid, Spain

27 CIBER Diabetes Y Enfermedades Metabólicas (CIBERDEM), Instituto de Salud Carlos III (ISCIII), Madrid, Spain

28 Department of Endocrinology, Institut d Investigacions Biomédiques August Pi Sunyer (IDIBAPS), Hospital Clinic, University of Barcelona, Barcelona, Spain 
29 Lipid Clinic, Department of Endocrinology and Nutrition, Institut d'Investigacions Biomèdiques August Pi Sunyer (IDIBAPS), Hospital Clínic, Barcelona, Spain

30 Department of Endocrinology and Nutrition, Hospital Fundación Jimenez Díaz, Instituto de Investigaciones

Biomédicas IISFJD, University Autonoma, Madrid, Spain
31 Nutritional Control of the Epigenome Group, IMDEA Food, CEI UAM + CSIC, Madrid, Spain

32 Department of Nutrition, Harvard T.H. Chan School of Public Health, Boston, MA, USA 